\title{
Common Origin of the Cerebellar Dual Somatotopic Areas Revealed by Tracking Embryonic Purkinje Cell Clusters with Birthdate Tagging
}

\author{
Khoa Tran-Anh, ${ }^{1}$ Jingyun Zhang, ${ }^{1}$ Viet Tuan Nguyen-Minh, ${ }^{1}{ }^{-}$Hirofumi Fujita, ${ }^{1,3}$ Tatsumi Hirata, ${ }^{4}$ and \\ (-)Izumi Sugihara,
}

https://doi.org/10.1523/ENEURO.0251-20.2020

${ }^{1}$ Department of Systems Neurophysiology, Graduate School of Medical and Dental Sciences, Tokyo Medical and Dental University, Bunkyo-ku, Tokyo 113-8519, Japan, ${ }^{2}$ Center for Brain Integration Research, Tokyo Medical and Dental University, Bunkyo-ku, Tokyo 113-8519, Japan, ${ }^{3}$ Department of Otolaryngology-Head and Neck Surgery, Johns Hopkins University, School of Medicine, Baltimore, Maryland 21205, U.S.A., and ${ }^{4}$ Brain Function Lab, National Institute of Genetics, Yata, Mishima-shi, Shizuoka-ken 411-8540, Japan

\section{Visual Abstract}
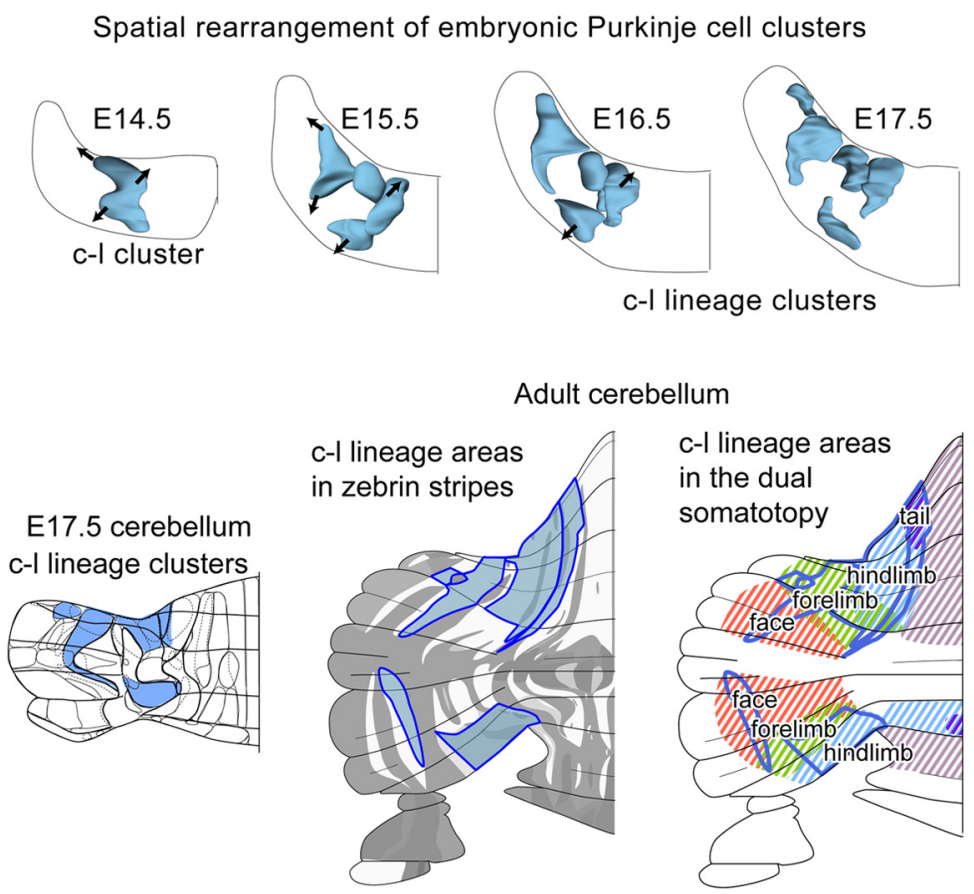

Left side of the cerebellum is shown in panels

One of the notable characteristics of the functional localization in the cerebellar cortex is the dual representation of the body (somatotopy) on its anterior-posterior axis. This somatotopy is conspicuous in the C1/C3 module, which is demarcated as the multiple zebrin-negative and weekly-positive stripes in dual paravermal areas in anterior and posterior lobules within the cerebellar compartments. In this report, we describe the early formation process of the cerebellar compartmentalization, particularly in the C1/C3 module. As developing PCs guide formation of the module-specific proper neuronal circuits in the cerebellum, we hypothesized that 
the rearrangement of embryonic Purkinje cell (PC) clusters shapes the adult cerebellar compartmentalization. By identifying PC clusters with immunostaining of marker molecules and genetical birthdate-tagging with Neurog2-CreER (G2A) mice, we clarified the three-dimensional spatial organization of the PC clusters and tracked the lineage relationships among the PC clusters from embryonic day 14.5 (E14.5) till E17.5. The number of recognized clusters increased from 9 at E14.5 to 37 at E17.5. Among E14.5 PC clusters, the c-I (central-lateral) cluster which lacked E10.5-born PCs divided into six c-I lineage clusters. They separately migrated underneath other clusters and positioned far apart mediolaterally as well as rostrocaudally by E17.5. They were eventually transformed mainly into multiple separate zebrin-negative and weakly-positive stripes, which together configured the adult $\mathrm{C} 1 / \mathrm{C} 3$ module, in the anterior and posterior paravermal lobules. The results indicate that the spatial rearrangement of embryonic PC clusters is involved in forming the dual somatotopic areas in the adult mouse paravermal cerebellar cortex.

Key words: cerebellum; compartmentalization; Purkinje cell; Purkinje cell cluster; somatosensory; somatotopic representation

\section{Introduction}

Representation of the somatotopy is deeply involved in the motor control function in the cerebellum (Manni and Petrosini, 2004). Electrophysiological and neuroimaging studies have shown dual somatotopic areas in the anterior and posterior cerebellar lobules which is one of the noticeable features of the functional localization of the cerebellar cortex of humans and other mammals (Fig. 1A; Thickbroom et al., 2003; Manni and Petrosini, 2004).

Morphologically, the cerebellar cortex is organized by multiple longitudinal striped subdivisions. Two types of mutually linked subdivisions, 1) modules and 2) molecular compartments, have been identified. The modules have been defined by the topographic connections of PC axons and climbing fiber axons (Fig. 1B; Voogd and Glickstein, 1998; Apps and Hawkes, 2009; Cerminara et al., 2013; Fujita and Sugihara, 2013; Ruigrok et al., 2015), whereas the molecular compartments have been defined by the arrangement of Purkinje cells (PCs) that show heterogeneous expression of marker molecules such as zebrin II or aldolase C (Fig. 1C; Brochu et al., 1990; Voogd and Glickstein,

Received June 12, 2020; accepted September 30, 2020; First published October 14, 2020.

Authors have no competing interests to declare.

Author contributions: K.T.-A. and I.S. designed research; K.T.-A., J.Z., V.T.N.-M., H.F., T.H., and I.S. performed research; K.T.-A., J.Z., V.T.N.-M., H.F., and I.S. analyzed data; K.T.-A., H.F., and I.S. wrote the paper; T.H. and I.S. contributed unpublished reagents/analytic tools.

Japan Society for the Promotion of Science (JSPS) [19K06919]; Japan Society for the Promotion of Science (JSPS) [20H03345]

Acknowledgments: This study was supported by Grant-in-Aid for Scientific Research (KAKENHI) from the Japan Society for the Promotion of Science (19K06919 to I.S., $20 \mathrm{H} 03345$ to T.H.) and ROIS Challenging Exploratory Research Projects for the Future Grant to T.H. The authors thank Dr. Gideon Sarpong and Mr. Richard Nana Abankwah Owusu Mensah for proofreading the manuscript. The authors thank Dr. Yuichi Ono of KAN Research Institute Inc. for providing us with anti-Corl2 antibody.

Corresponding should be addressed to Izumi Sugihara at isugihara.phy1@ tmd.ac.jp.

https://doi.org/10.1523/ENEURO.0251-20.2020

Copyright @ 2020 Tran-Anh et al.

This is an open-access article distributed under the terms of the Creative Commons Attribution 4.0 International license, which permits unrestricted use, distribution and reproduction in any medium provided that the original work is properly attributed.
1998; Sugihara and Shinoda, 2004; Sillitoe and Joyner, 2007; Fujita et al., 2014). The somatotopic representation is most clearly seen in the paravermal area in anterior and posterior lobules, in which zebrin-negative and -faintly-positive stripes (identical to the $\mathrm{C} 1 / \mathrm{C} 3$ module; Fig. $1 B, C$ ) occupy substantial proportions of the cerebellar cortex. Both the anterior and posterior parts of this area are topographically innervated by the climbing fiber axons originating from the dorsal accessory olive and project to the anterior interposed nucleus (Ekerot et al., 1997; Cerminara et al., 2013; Ruigrok et al., 2015; Low et al., 2018) to be involved in the control of fine body movements such as grasping and limb cutaneous reflexes in the cat (Horn et al., 2010) and rat (Pijpers et al., 2008). Because the C1/C3 module represents the main part of the cerebellar somatotopic area as mentioned above, the anteroposterior separation of the $\mathrm{C} 1 / \mathrm{C} 3$ module (Fig. 1B) may be the anatomical correlate for the anteroposterior dual representation of somatotopy observed in animal and human cerebellums (Snider, 1950; Stoodley et al., 2012; Guell et al., 2018).

PCs are born in the period between embryonic day 10.5 (E10.5) and E12.5 in the ventricular zone (Hashimoto and Mikoshiba, 2003) and form the main body of the immature cerebellum by E14.5 in mice (Goffinet, 1983). At E14.5, some eight heterogeneous subsets of PCs are arranged in clustered compartments as observed by molecular marker labeling (Vibulyaseck et al., 2017) or genetic profiling (Wizeman et al., 2019). At E17.5, the number of heterogeneous populations of PCs increases to about 50, which are arranged into clusters separated by PC-free gaps (Fig. 1E; Korneliussen, 1968; Altman and Bayer, 1985; Smeyne et al., 1991; Oberdick et al., 1993; Millen et al., 1995; Larouche et al., 2006; Wilson et al., 2011; Fujita et al., 2012; Carter et al., 2018; Wizeman et al., 2019). Each of the E17.5 clusters develops directly into an individual adult PC stripe in the postnatal period (Fig. 1D; Sillitoe et al., 2009; Namba et al., 2011; Fujita et al., 2012). Therefore, we hypothesized that the rearrangement of embryonic PC clusters is essential in shaping the compartmental organization of the adult cerebellar cortex which includes its modular organization and the dual somatotopic areas. However, accurate spatial tracking of lineages of all E14.5 clusters would be required to test this hypothesis. 

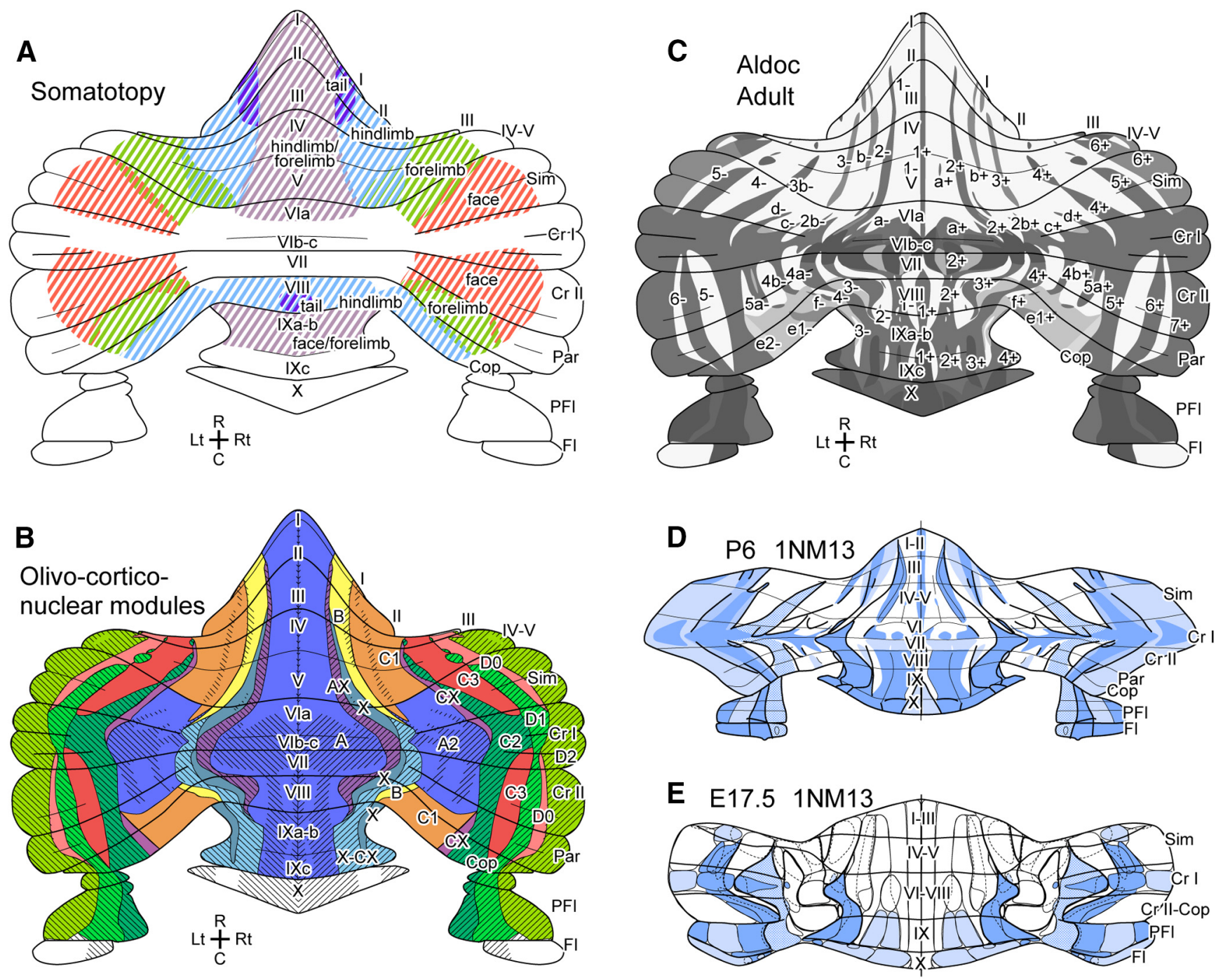

Figure 1. Introductory schematic drawings of the somatotopy and compartments mapped in the unfolded scheme of the mouse cerebellar cortex. A, Somatotopy mainly based on mapping of mossy fiber terminal response (Welker, 1987) and labeled spinocerebellar and cuneocerebellar projections in rodents (Quy et al., 2011; Luo et al., 2018, 2020). B. Olivocorticonuclear modules (colored areas) defined by the topographic axonal projections between subareas of the inferior olive, cerebellar nuclei and cerebellar cortex (A, AX, A2, B, CX, X, X-CX, C1, C2, C3, D0, D1 and D2 modules; Voogd and Glickstein, 1998; Sugihara and Shinoda, 2004; Ruigrok et al., 2015). Shadowed areas belong to aldolase C-positive stripes. C, Stripes defined by aldolase C expression pattern in Purkinje cells (Fujita et al., 2014; Sarpong et al., 2018). D and $\boldsymbol{E}$, Mapping of clusters of PCs at E14.5 and immature stripes of PCs at P6 based on Fujita et al. (2012). Blue areas indicate particular clusters or immature stripes that express lacZ in $1 \mathrm{NM} 13$ transgenic mice, which often overlap with zebrin stripes. Abbreviations, c-l, c-m, d, dl, I, m, ml, rdl, vl, names of E14.5 clusters; C, caudal; D, dorsal; L, lateral; M, medial; R, rostral. V, ventral.

Each striped compartment in the adult cerebellar cortex contains PCs generated at particular timing (Hashimoto and Mikoshiba, 2003; Namba et al., 2011; Zhang et al., 2020). Therefore, birthdate-specific labeling of PCs can be a useful technique to track the cerebellar compartmentalization. The CreER-LoxP system that targets the asc/1 gene, which is transiently expressed at the time of neuronal differentiation, can label neurons that are born at the time of tamoxifen injection (Sudarov et al., 2011). We used a similar birthdate-tagging system (G2A mouse line, Hirata et al., 2019; Zhang et al., 2020) that targets the Neurog2 gene, which is expressed in neurons including PCs (Zordan et al., 2008), when the neuronal progenitors start differentiating (Florio et al., 2012).
By combining birthdate-specific labeling and molecular marker labeling of PCs (Minaki et al., 2008; Fujita et al., 2012), we tracked the migration and division of all embryonic PC clusters from E14.5 to E17.5 to clarify the spatial development of the cerebellar compartmentalization. We then focused on the lineage of a particular E14.5 cluster, the fate of which was crucial to test the above hypothesis.

\section{Materials and Methods}

\section{Ethics statements}

Experimental protocols were approved by the Animal Care and Use Committee (A2019-187A, A2018-148A, A2017-060C4) and Gene Recombination Experiment 
Safety Committee (G2019-020A, 2017-040A, 2012064C4) of Tokyo Medical and Dental University.

\section{Animals}

Mice were bred and reared in a 12-12-hour lightdark cycled condition in the animal facility of the university with freely available food and water. Wildtype embryo samples were obtained by mating $\mathrm{B} 6 \mathrm{C} 3 \mathrm{~F} 1$ males and females. The C57BL/6N-Tg(Neurogenin2-CreER) mouse strain (G2A, deposited at RIKEN BDRAccession No. CDB0512T-1, http://www2.clst.riken.jp/arg/TG\%20 mutant\%20mice\%20list.html, Hirata et al., 2019) has the transgene, in which CreER gene has been inserted into the downstream side of the enhancer region of neurogenin2 gene (Neurog2), presumptively on the $\mathrm{Y}$ chromosome (Hirata et al., 2019). Since the CreER is expressed in differentiating PCs after the last mitosis under the Neurog2 enhancer (Florio et al., 2012), administration of tamoxifen, a ligand of the estrogen receptor, produces Cre activity in cells in which Neurog2 is expressed in G2A mice. In B6.Cg-

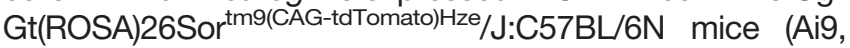
The Jackson Laboratory, https://www.jax.org/strain/007), Cre activity produces a persistent tdTomato expression in cells. Male heterozygous G2A mice were crossed with female homozygous Ai9 mice to produce G2A::Ai9 embryos. The day when the vaginal plug was detected was designated as E0.5. Tamoxifen (T5648-1G, Sigma, St. Louis, MO, U.S.A.) was dissolved in corn oil (9 mmole/l, 032-17016, Wako, Wako Pure Chemical Industries, Ltd., Osaka, Japan) and injected intraperitoneally $(2.25 \mu \mathrm{mole} / \mathrm{mouse})$ one time to the pregnant female at noon, 10, 11 or 12 days after the plug detection (at E10.5, E11.5, E12.5). In AldocV mice (MGI:5620954, Fujita et al., 2014), aldolase C (zebrin II) stripes are labeled by a mutated green fluorescent protein, Venus. We produced double-homozygous Ai9::AldocV mice. An Ai9::AldocV double-homozygous female was crossed with a G2A heterozygous male (Zhang et al., 2020). Tamoxifen was injected into the pregnant female as above. E19.5 embryos were obtained by Caesarean section from pregnant Ai9::AldocV double homozygous females which were sacrificed beforehand by cervical dislocation. Male pups (G2A::Ai9::AldocV heterozygous hybrid mice) were reared by a stepmother and perfused at postnatal day 42 .

\section{Histological procedures}

A Caesarean section was performed on pregnant Ai9 females anesthetized with an intramuscular injection of pentobarbital sodium $(0.1 \mathrm{mg} / \mathrm{g}$ body weight) and xylazine ( $0.005 \mathrm{mg} / \mathrm{g}$ body weight) to obtain E14.5-E17.5 embryos at noon. Embryos were perfused transcardially with phosphate-buffered saline (PBS, pH 7.4) with heparin sulfate $(0.1 \%)$, and then with $4 \%$ paraformaldehyde. The anesthetized female was sacrificed by cervical dislocation after removing embryos. The embryo brains were dissected in chilled $4 \%$ paraformaldehyde and kept in $4 \%$ paraformaldehyde for post-fixation and then soaked in sucrose solution ( $30 \%$ with $0.05 \mathrm{M}$ phosphate buffer, $\mathrm{pH}$
7.4) for two days. Among embryo samples obtained from pregnant Ai9 female, ones that showed patterned red fluorescence reporter expression in the brain were regarded as G2A::Ai9 heterozygous hybrid. Brain samples were stored in the deep freezer until sectioning.

G2A::Ai9::AldocV heterozygous hybrid mice were anesthetized with an intramuscular injection of an overdose of pentobarbital sodium $(0.18 \mathrm{mg} / \mathrm{g}$ body weight) and xylazine $(0.009 \mathrm{mg} / \mathrm{g}$ body weight) at postnatal day 42 . They were perfused transcardially with PBS with $0.1 \%$ heparin sulfate, and then with $4 \%$ paraformaldehyde. The skull was kept in $4 \%$ paraformaldehyde for post-fixation overnight. The brain was dissected and soaked in sucrose solution for two days. Brain samples were stored in the deep freezer until sectioning.

Embryo G2A::Ai9 brains were coated with gelatin solution $(10 \%$ gelatin, $10 \%$ sucrose in $10 \mathrm{mM}$ phosphate buffer, $\left.\mathrm{pH} 7.4,32^{\circ} \mathrm{C}\right)$. The gelatin block was hardened by chilling and then soaked overnight in fixative with a high sucrose content (4\% paraformaldehyde, $30 \%$ sucrose in $0.05 \mathrm{M}$ phosphate buffer, $\mathrm{pH}$ 7.4). Complete sets of serial sections were cut coronally, horizontally and sagittally using freezing microtome at a thickness of $40 \mu \mathrm{m}$. The ventral surface of the medulla was regarded as the horizontal plane. After washing in PBS and PBS with $0.12 \%$ Triton X-100 (PBST), each complete set of sections was processed for immunostaining. Floating sections were incubated on a shaker with a mixture of two or three primary antibodies produced in different host animal species in PBST plus $2 \%$ normal donkey serum for 48 hours at $4^{\circ}$ C. Goat anti-EphA4 (R\&D Systems), goat anti-FoxP2 (Everest Biotech), rabbit anti-Corl2 (provided by Dr. Ono at KAN Research Institute) and rat anti-OL-protocadherin (Millipore) are the primary antibodies used in the majority of experiments. Rabbit anti-FoxP2 antibody (Abgent) was used in combination with the goat primary antibody. The specificity of the above antibodies has been described (Vibulyaseck et al., 2017). In some experiments, mouse anti-Calbindin-D28k (Sigma-Aldrich) and rabbit antiCalbindin-D28k (AnaSpec) antibodies were also used (Table 1). The sections were then incubated with a mixture of appropriate two or three secondary antibodies that were conjugated with fluorescent tags (Table 1). Some sections were counterstained with 4', 6-diamidino-2-phenylindole dihydrochloride (DAPI; 1:3,000; D212, Dojindo, Mashiki, Kumamoto, Japan). Finally, these sections were mounted on glass slides, dried, coverslipped with watersoluble mounting medium (CC mount, Sigma C936830ML).

Postnatal G2A::Ai9::AldocV brains were embedded in gelatin, and cut coronally into serial sections at a thickness of $50 \mu \mathrm{m}$. The complete sets of sections were mounted on glass slides, dried, coverslipped with watersoluble mounting medium (CC mount).

\section{Acquisition of digital images}

Fluorescent images were digitized using a cooled color CCD camera (AxioCam1Cm1, Zeiss, Oberkochen, Germany) attached to a fluorescent microscope (Axiolmager.Z2, Zeiss) in 12-bit gray-scale with appropriate filter sets. To digitize a 
Table 1: Antibodies used in the study

\begin{tabular}{|c|c|c|c|}
\hline & Antigen & $\begin{array}{l}\text { Manufacturer, species, mono- or } \\
\text { polyclonal, catalog or lot No., RRID }\end{array}$ & Concentration \\
\hline \multirow[t]{6}{*}{$\begin{array}{l}\text { Primary } \\
\text { Antibodies }\end{array}$} & Corl2 & $\begin{array}{l}\text { Dr. Yuichi Ono (KAN Research Institute), rabbit polyclonal, } \\
\text { affinity-purified }\end{array}$ & $1: 350$ \\
\hline & EphA4 & $\begin{array}{l}\text { R\&D Systems, goat polyclonal, Cat\# AF641, Lot } \\
\text { \#BVX0308091 }\end{array}$ & $1: 1000$ \\
\hline & FoxP2 & $\begin{array}{l}\text { Everest Biotech (Oxfordshire, UK), goat polyclonal, Cat\# } \\
\text { EB05226, Lot \# 160409, RRID: AB_2107112 }\end{array}$ & $1: 5000$ \\
\hline & FoxP2 & $\begin{array}{l}\text { Abgent, rabbit polyclonal, Cat\# AP5753b, Lot } \\
\text { \#SA100916AA, RRID: AB_10818782 }\end{array}$ & $1: 1000$ \\
\hline & Pcdh10 & $\begin{array}{l}\text { Millipore, rat monoclonal, clone 5G10, Cat\# MABT20, Lot \# } \\
\text { NRG1759424, RRID:AB_10807416 }\end{array}$ & $1: 1600$ \\
\hline & Calbindin D28 & Sigma, mouse monoclonal, Cat\# $175651 \mathrm{C} 8666$ & $1: 500$ \\
\hline \multirow{11}{*}{$\begin{array}{l}\text { Secondary } \\
\text { Antibodies }\end{array}$} & Anti-Goat lgG, Alexa Fluor 488 & Jackson ImmunoResearch, donkey, Cat\# 705-545-147 & $1: 400$ \\
\hline & Anti-Goat IgG, Alexa Fluor 680 & Jackson ImmunoResearch, donkey, Cat\# 705-625-147 & $1: 400$ \\
\hline & Anti-Rabbit IgG, Alexa Fluor 405 & abcam, donkey, Cat\# 175651 & $1: 500$ \\
\hline & Anti-Rabbit IgG, Alexa Fluor 488 & Jackson ImmunoResearch, donkey, Cat\# 711-545-152 & $1: 400$ \\
\hline & Anti-Rabbit IgG, Alexa Fluor 647 & Jackson ImmunoResearch, donkey, Cat\# 711-605-152 & $1: 400$ \\
\hline & Anti-Rabbit IgG, Alexa Fluor 594 & Jackson ImmunoResearch, donkey, Cat\# 711-585-152 & $1: 400$ \\
\hline & Anti-Rabbit IgG, Teas Red & Jackson ImmunoResearch, donkey, Cat\# 711-075-152 & $1: 200$ \\
\hline & Anti-Rat IgG, DyLight 594 & Jackson ImmunoResearch, donkey, Cat\# 712-515-153 & $1: 200$ \\
\hline & Anti-Rat lgG, Alexa Fluor 647 & Jackson ImmunoResearch, donkey, Cat\# 705-605-150 & $1: 400$ \\
\hline & Anti-Rat lgG, Alexa Fluor 680 & abcam, donkey, Cat\# 175777 & $1: 500$ \\
\hline & Anti-Mouse IgG, Alexa Fluor 647 & Jackson ImmunoResearch, donkey, Cat\# 715-605-150 & $1: 400$ \\
\hline
\end{tabular}

section of the cerebellum, 2.5X objective and tiling function of the software to control digitizing (Zen 2 Pro, Zeiss) was used. Images of all serial sections of a brain were obtained with the same exposure and gain parameters. Images were adjusted in contrast and brightness and assembled using a software (ZEN 2 Pro, Zeiss and Photoshop 7, Adobe, San Jose, CA, USA). High magnification confocal images were taken with a 63X objective lens and appropriate filters and laser light sources attached to the confocal microscope (TCS SP8, Leica, Wetzlar, Germany). Images were adjusted in contrast and brightness and assembled using software (Las X, Leica). A combination of pseudo-colors was applied to fluorescent and confocal images in figures. Photographs were assembled using Photoshop and Illustrator software (Adobe). Digital enhancements were applied to entire images and no manipulations were applied other than contrast or brightness.

\section{Three-dimensional reconstruction of Purkinje cell clusters}

Three-dimensional (3D) models of PC clusters were reconstructed through two steps: 1) two dimensional (2D) drawings of contours of identified PC clusters, and 2) 3D surface modeling with these 2D drawings. Digital images of serial sections were placed in individual layers of $2 \mathrm{D}$ graphic software (Adobe Illustrator 10). Their positions and orientations were then adjusted by superimposing them on each other while referring to landmark structures such as the midline, the cerebellar surface and major labeled areas. The cerebellar surface and contour of groups of Purkinje cell subsets and marker-labeled areas (i.e. PC cluster) were drawn using curve tools of Illustrator in all sections. Distribution patterns of PCs and PC-free gaps, and expression patterns of PC markers were systematically observed in this procedure. Cerebellar nuclear areas identified by the lack of Corl2 signal were excluded from the reconstruction. After the identification of PC clusters, all drawings in sections of coronal, horizontal and sagittal planes were imported into 3D graphics software (Rhinoceros 4, Robert McNeel \& Associates, Seattle, WA, USA), with the z-axis position adjusted for each section. The 3D drawings of cerebellar structures obtained from brain samples with different cutting orientations were matched and compared with one another to identify structures. The 3D surface reconstructions were made from a set of coronal section drawings by using the 'loft' command in Rhinoceros (Fujita et al., 2012). Cerebellar fissures were reconstructed in the 3D space from aligned drawings from sagittal sections.

\section{Definition of the relative position of a coronal plane within the cerebellum}

The relative position of a section in the whole extent of the cerebellum was defined using percentages as described previously (Vibulyaseck et al., 2017). In short, the position of the most caudal section of the coronal sections in which the cerebellum first appeared was defined as $0 \%$, whereas the position of the most rostral section in which the cerebellum remained was defined as $100 \%$. The position of other sections was obtained by linear interpolation. In the case of horizontal sections, the most dorsal section was defined as $0 \%$, while the most ventral section in which the cerebellum remained was defined as $100 \%$.

\section{Results}

\section{Spatial organization of PC clusters in the embryonic cerebellums at E14.5, E15.5, E16.5, and E17.5}

Although embryonic PC clusters were suggested to be the direct origin of adult cerebellar compartments (Fujita 
et al., 2012), the development of embryonic PC clusters has not been fully clarified before E17.5 except for the Pcdh10-positive areas which have been tracked in our previous study (Vibulyaseck et al., 2017). In the first set of experiments in the present study, we identified PC clusters in the entire cerebellum at a 1-day interval from E14.5 to E17.5. The distribution of PCs was analyzed by examining the expression of particular molecular markers and PC-free gaps in serial coronal, horizontal and sagittal sections in 22 wild-type B6C3F1 mouse embryos (E14.5, $\mathrm{n}=7$; E15.5, $\mathrm{n}=5$, E16.5, $\mathrm{n}=5$, E17.5, $\mathrm{n}=5$ ). Expression of FoxP2 (relatively specific PC marker, Fujita et al., 2012; Vibulyaseck et al., 2017), Corl2 (also known as Skor2, specific PC marker, Minaki et al., 2008; Vibulyaseck et al., 2017), Pcdh10 (PC cluster marker, Fujita et al., 2012; Vibulyaseck et al., 2017) and EphA4 (PC cluster marker expressed in PCs and afferent axons, Fujita et al., 2012; Vibulyaseck et al., 2017) were immunohistochemically revealed.

We compared the labeling pattern between left and right sides and among different samples cut in coronal, horizontal and sagittal sections at each embryonic date. Interindividual variations, such as those in the marker expression level, shape, size, and positional relationships of clusters were small. Thus, we comprehensively identified PC clusters at E14.5 (Fig. 2), E15.5 (Fig. 3), E16.5 (Fig. 4), and E17.5 (Fig. 5), and confirmed previously identified PC clusters at E14.5 (Vibulyaseck et al., 2017) and E17.5 (Fujita et al., 2012). We then reconstructed identified PC clusters of E14.5, E15.5 and E16.5 cerebellums in the three-dimensional space (Figs. $2 \mathrm{I}, 3 \mathrm{H}, 4 \mathrm{~J}$ ) primarily based on images of immunostaining on one side of serial coronal sections. For those of E17.5, the previously published reconstruction (Fujita et al., 2012) was incorporated with some revisions (see below; Fig. 5J).

At E14.5, nine PC clusters-termed medial, dorsal, central-medial, central-lateral, mid-lateral, lateral, dorsolateral, rostrodorsolateral, and ventrolateral (m, d, c-m, c-l, ml, l, dl, rdl, and vl; based on Vibulyaseck et al., 2017)-were arranged in column-shaped elongations in the rostrocaudal direction at various mediolateral and dorsoventral levels (Fig. 2/). A previously designated Pcdh10-positive cluster, termed "c" (Vibulyaseck et al., 2017), was revised here: it was divided into the central-medial (c-m) and central-lateral (c-I) clusters because the expression of Corl2 was stronger in the $\mathrm{c}-\mathrm{m}$ than in the $\mathrm{c}-\mathrm{I}$ (Fig. 2A-C). The distribution of these nine clusters was remarkably consistent with that of the unbiasedly classified groups of PCs via single-cell RNAseq (Wizeman et al., 2019; see Discussion). These clusters, thus, may reflect fundamental molecular distinctions in PCs at E14.5.

The number of PC clusters increased from 9 at E14.5 (Fig. 2), to 18 at E15.5 (Fig. 3), and to 28 at E16.5 (Fig. 4). The number increased presumably because 1) new PCfree gaps appeared inside a cluster, and/or 2) a part of a cluster changed in molecular expression from the other part of the same cluster. Furthermore, some divided clusters seemed to migrate separately. At E17.5, PC clusters were narrow in the mediolateral direction but often extended rostrocaudally (Iongitudinally) across immature lobules, somewhat resembling adult striped compartments, although they were not yet arranged in a single layer but in multiple layers, shallow or deep from the cerebellar surface (Fig. 5; Fujita et al., 2012).

Although E17.5 cerebellum contains 54 clusters identified with detailed analyses (Fujita et al., 2012), this study focused on the more qualitative distinction of clusters to facilitate analyses and simplify description. Namely, neighboring clusters that had only slightly different molecular expression profiles and/or not clearly separated from one another by intercalating PC-free gaps were combined. For example, our cluster vp1-2 includes clusters vp1 and vp2 of Fujita et al. (2012). We combined 11 sets of two or three neighboring E17.5 clusters into single clusters, resulting in a total of 37 clusters in place of 54 . We adopted the nomenclature (Table 3) from Fujita et al. (2012) to designate E17.5 clusters in the present study.

\section{Birthdate-specific labeling of PCs in the E14.5 cerebellum}

In the second set of experiments, we labeled PCs in the birthdate-specific way to identify the lineage of PC clusters. The G2A mouse line expressed tamoxifen-inducible Cre recombinase activity under the transcription control of proneural gene, neurogenin 2 (Neurog2). We crossed female Cre-reporter mice (Ai9) with male heterozygous G2A mice so that tamoxifen injection into the pregnant dam at a specific developmental stage of E10.5, E11.5 and E12.5 (designated as TM10.5, TM11.5 and TM12.5) enabled timed activation of Cre recombinase that initiates reporter (tdTomato) expression in PCs in a birthdate-specific way (Fig. 6A-D). G2A::Ai9 hybrid embryo brain samples were collected between E14.5 and E17.5 ( $n=23$ total: E14.5-TM10.5, n=1; E14.5-TM11.5, n=1; E14.5-TM12.5, $\mathrm{n}=1$; E15.5-TM10.5, $\mathrm{n}=2$; E15.5-TM11.5, $\mathrm{n}=1 ; \mathrm{E} 15.5-$ TM12.5, n=3; E16.5-TM10.5, n=3; E16.5-TM11.5, $\mathrm{n}=1$; E16.5-TM12.5, n=3; E17.5-TM10.5, $n=3 ; E 17.5-T M 11.5$, $\mathrm{n}=2$; E17.5-TM12.5, $\mathrm{n}=2$ ), cut into serial sections and immunostained for EphA4, Pcdh10, and either FoxP2 or Corl2. Combined labeling of EphA4 and Pcdh10 helped to recognize the clusters that were identified in the analyses described in the preceding section (Figs. 2-5). No clear difference was observed in the PC cluster organization between G2A::Ai9 mice with C57BL/6 background and wild type mice with B6C3F1 background.

Tamoxifen administration labeled neurons with tdTomato in the cerebellum of G2A::Ai9 mice (Fig. 6A-D). The labeling pattern was dependent on the timing of tamoxifen administration but consistent among cases that had the same administration timing. We first checked the specificity and efficiency of the labeling in the E14.5 cerebellum. All tdTomato-expressing cells $(100 \%)$ inside a PC cluster coexpressed Corl2 (Fig. 6F), in all mice $(n=23)$, indicating that tdTomato labeling was specific to PCs. Efficiency of the labeling was estimated by counting the number of tdTomato-expressing PCs among all PCs. Because the majority of PCs are born between E10.5 and E12.5, the sum of the ratios of the tdTomato-labeled PCs in the TM10.5, TM11.5, and TM12.5 cerebellums would become close to $100 \%$ if the efficiency is high. Indeed, in 
E14.5 Corl2 FoxP2 Pcdh10

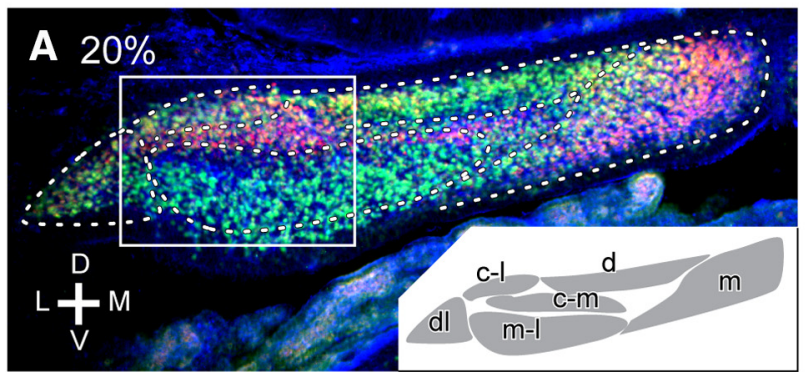

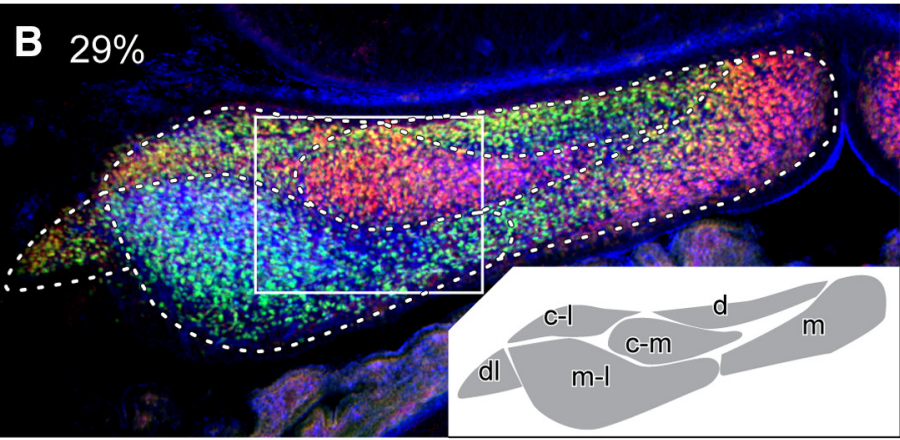
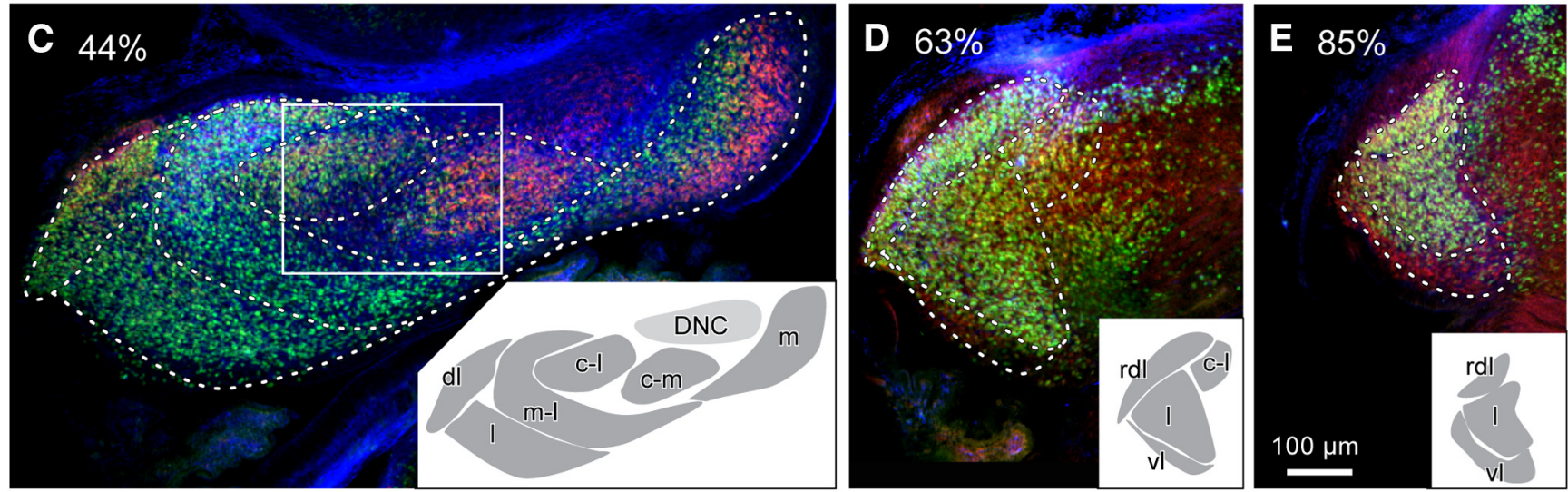
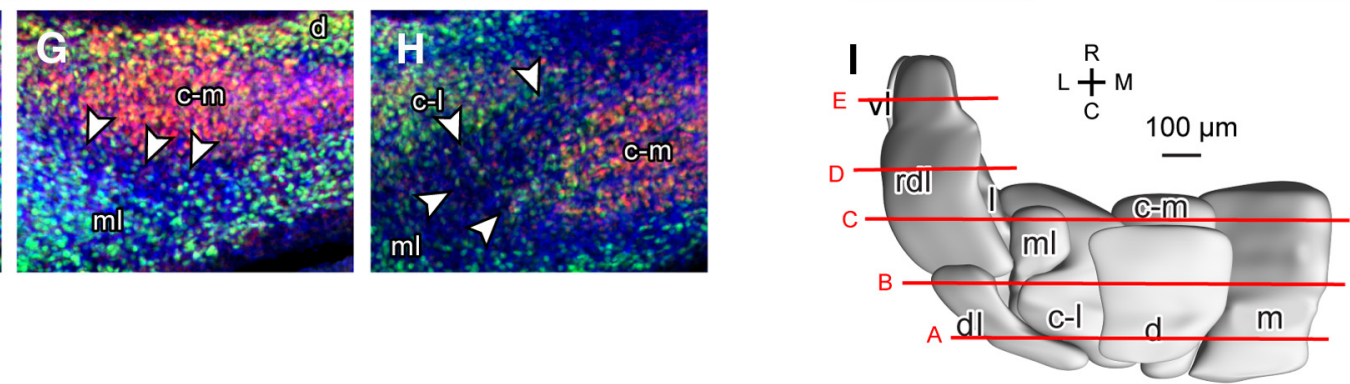

Figure 2. Purkinje cell clusters in coronal sections of the left E14.5 mouse cerebellum identified by marker expression profiles. $\boldsymbol{A}-\boldsymbol{E}$, Sections at different caudorostral levels indicated by percentile. Fluorescent signals of immunostaining for Corl 2 (red), Pcdh10 (blue) and FoxP2 (green) are merged. White dashed lines indicate the boundary of recognized clusters. Inset in each panel shows drawings of recognized clusters at half magnification. Squares indicate areas that are magnified. $\boldsymbol{F}-\boldsymbol{H}$, Magnified images showing PC-free gaps (arrowheads) between some clusters. I, Dorsal view of the 3D reconstruction of clusters. Red lines indicate the rostrocaudal level of the section in each panel. Scale bar in $\mathrm{E}(100 \mu \mathrm{m})$ applies to $\boldsymbol{A}-\boldsymbol{E}$. Scale bar in F $(100 \mu \mathrm{m})$ applies to $\boldsymbol{F}-\boldsymbol{H}$. Abbreviations, C-I, C-m, d, dl, I, m, ml, rdl, vl, names of E14.5 clusters; C, caudal; D, dorsal; DCN, deep cerebellar nucleus; L, lateral; M, medial; R, rostral.V, ventral.

the $10,000-\mu \mathrm{m}^{2}$ area of dense PC distribution within the $\mathrm{ml}$ cluster of TM10.5, TM11.5, and TM12.5 cerebellums $(\mathrm{n}=1$ each) at $\mathrm{E} 14.5,41.1 \%$ (44 PCs out of $107,44 / 107)$, $52.0 \%(52 / 100)$, and $4.7 \%$ (5/106) of PCs were tdTomatopositive, respectively, indicating an estimated efficiency of $97.8 \%$. A similar measurement in the c-I cluster showed $1.1 \%$ (1/89), 40.8\% (40/98) and 45.2\% (42/93) labeled PCs in TM10.5, TM11.5 and TM12.5 cerebellums $(n=1$ each), respectively, at E14.5, indicating an estimated efficiency of $87.1 \%$. The results indicated that the recombination is highly efficient as well as highly dependent on the timing of tamoxifen administration. Similar efficiency and timing-dependency have been also observed in PCs in the adult cerebellum (Zhang et al., 2020). Thus, the PCs labeled by the tamoxifen injection on a specific timing were designated as "E10.5-born" etc. and the area or cluster in which about $1 \%$ of PC were labeled with tamoxifen injection at E10.5 was designated as "E10.5-PCsparse".

We then examined the distribution of E10.5-born, E11.5-born and E12.5-born PCs in the PC clusters, which were identified as described in the preceding section, in the E14.5 cerebellum. (Fig. $6 A-D$ and Table 2). E10.5born PCs were observed densely in the $\mathrm{d}$, $\mathrm{ml}$, and rdl clusters, moderately or sparsely in the $\mathrm{c}-\mathrm{m}, \mathrm{l}, \mathrm{dl}$, and $\mathrm{vl}$ clusters (Fig. 6A1, B1, C1, D1). However, almost none of the E10.5-born PCs were observed in the $\mathrm{m}$ and $\mathrm{c}-\mathrm{I}$ clusters (blue asterisks in Fig. 6A1, B1, C1). E11.5-born PCs were observed in all clusters (Fig. 6A2, B2, C2, D2). They were more densely distributed in the $\mathrm{d}, \mathrm{ml}$, and rdl clusters than in other clusters, and were absent in the medial part of the $\mathrm{m}$ cluster. E12.5-born PCs distributed densely in the $\mathrm{m}$ 
E15.5 Corl2 FoxP2 Pcdh10
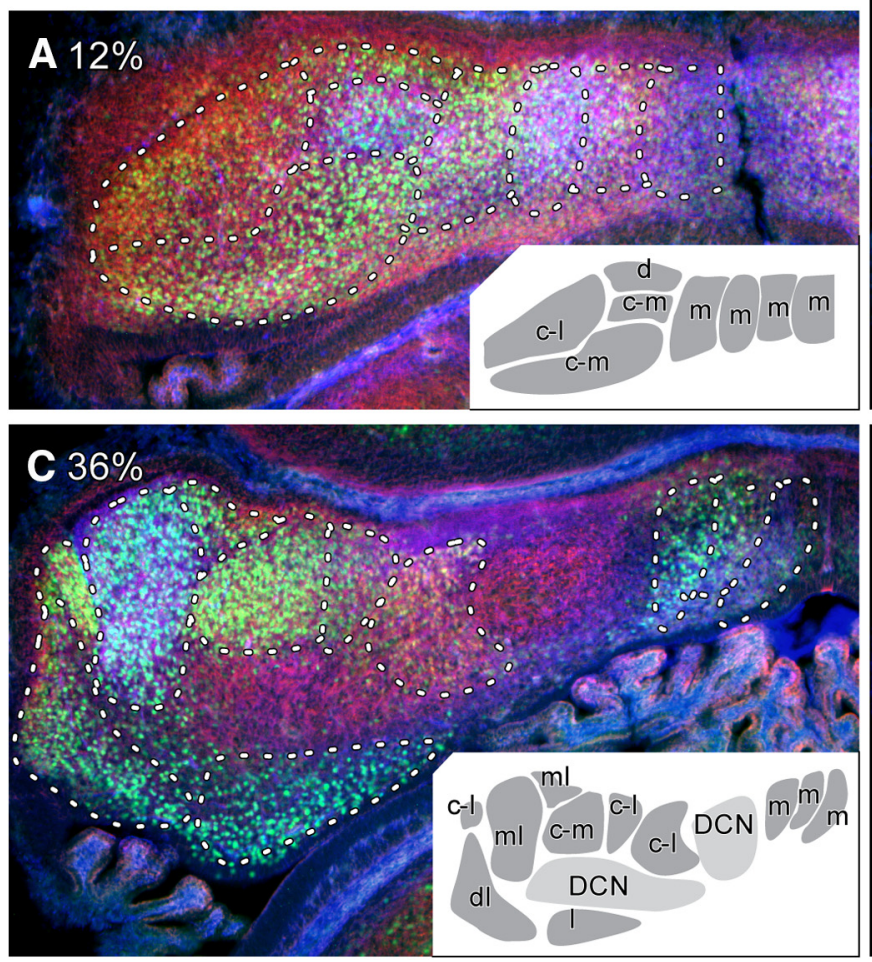
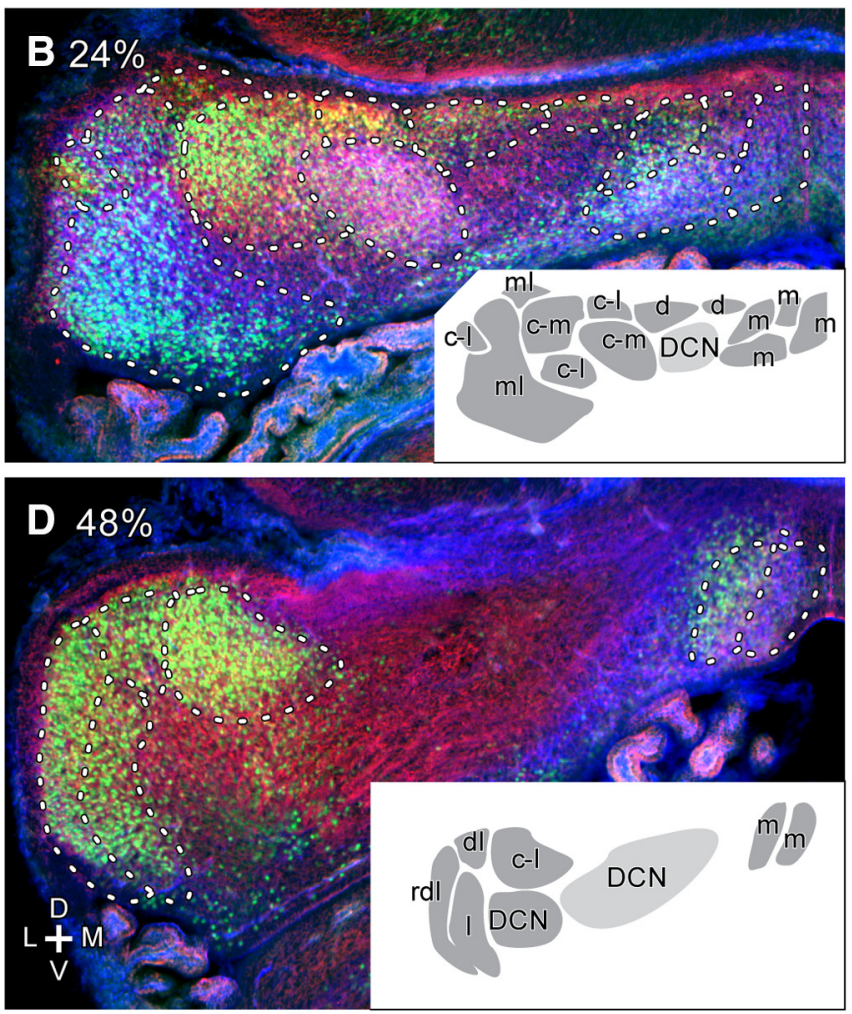
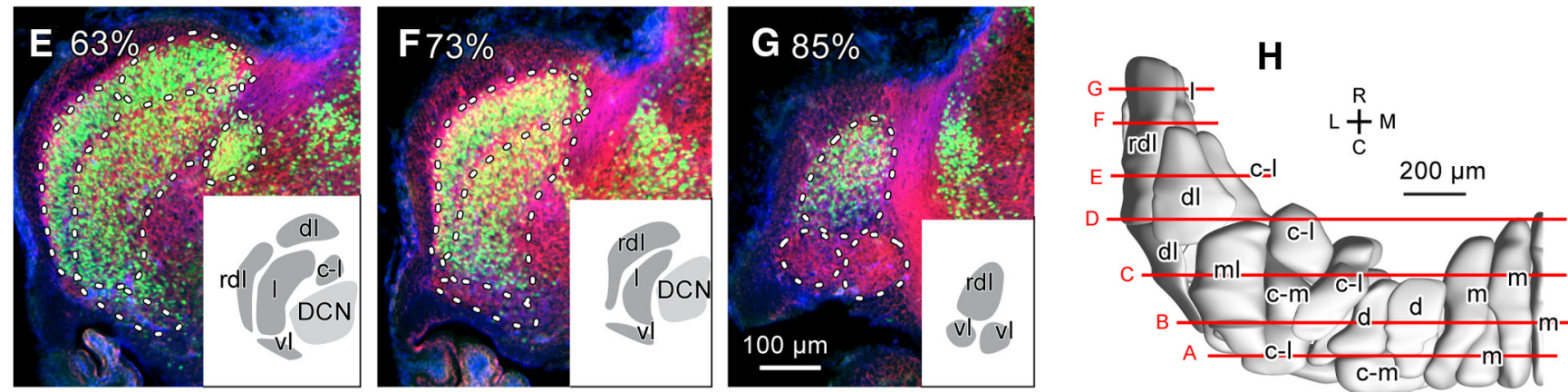

Figure 3. Purkinje cell clusters in coronal sections of the left E15.5 mouse cerebellum identified by marker expression profiles. A-G, Sections at different caudorostral levels indicated by percentile. Fluorescent signals of immunostaining for Corl 2 (red), Pcdh10 (blue) and FoxP2 (green) are merged. White dashed lines indicate the boundary of recognized clusters. Inset in each panel shows drawings of recognized clusters at half magnification. Names of each cluster were given later based on the lineage analysis (c.f. Fig. 7). $\boldsymbol{H}$, Dorsal view of the 3D reconstruction of clusters. Red lines indicate the rostrocaudal level of the section in each panel. Scale bar in $\mathrm{G}(100 \mu \mathrm{m})$ applies to panels $\boldsymbol{A - G}$. Abbreviations, c-l, c-m, d, dl, I, m, ml, rdl, vl, names of the lineage of clusters; C, caudal; $\mathrm{D}$, dorsal; DCN, deep cerebellar nucleus; L, lateral; M, medial; R, rostral.V, ventral.

cluster and moderately in the c-m, c-I, I, and vl clusters (Fig. 6A3, B3, C3, D3), but rarely contributed to either the $\mathrm{d}, \mathrm{ml}, \mathrm{dl}$ or rdl clusters (orange asterisks in Fig. 6A3, B3, C3, D3). This observation was further quantified by measuring the fluorescence signal intensity, which was supposed to be approximately linearly related to the density of labeled PCs, in each cluster, in digital images of sections of TM10.5, TM11.5 and TM12.5 cerebellums (Fig. $6 E)$. The labeling densities of the $\mathrm{c}-\mathrm{I}$ and $\mathrm{m}$ clusters were near 0 , lower than those of other clusters in the TM10.5 cerebellum. but increased to higher levels in the TM11.5 and TM 12.5 cerebellums. On the contrary, the labeling densities of the $\mathrm{ml}, \mathrm{d}$, and rdl clusters were higher than those of other clusters in the TM10.5 and TM11.5 cerebellums, but decreased to the level near 0 , lower than those of other clusters in the TM12.5 cerebellum (Fig. 6E).

Within each cluster excepting the $\mathrm{m}$ cluster, PCs that were born on a particular birthdate appeared to distribute randomly. Boundaries of different labeling density areas matched with the boundaries of the clusters defined by marker expression profiles and PC-free gaps in the TM10.5, TM11.5 and TM12.5 cerebellums (Fig. 6A1-A3, center subpanels, asterisks). Each E14.5 cluster was composed of the PCs of two or more particular birthdates (Table 2). 


\section{E16.5 EphA4 FoxP2 Pcdh10}
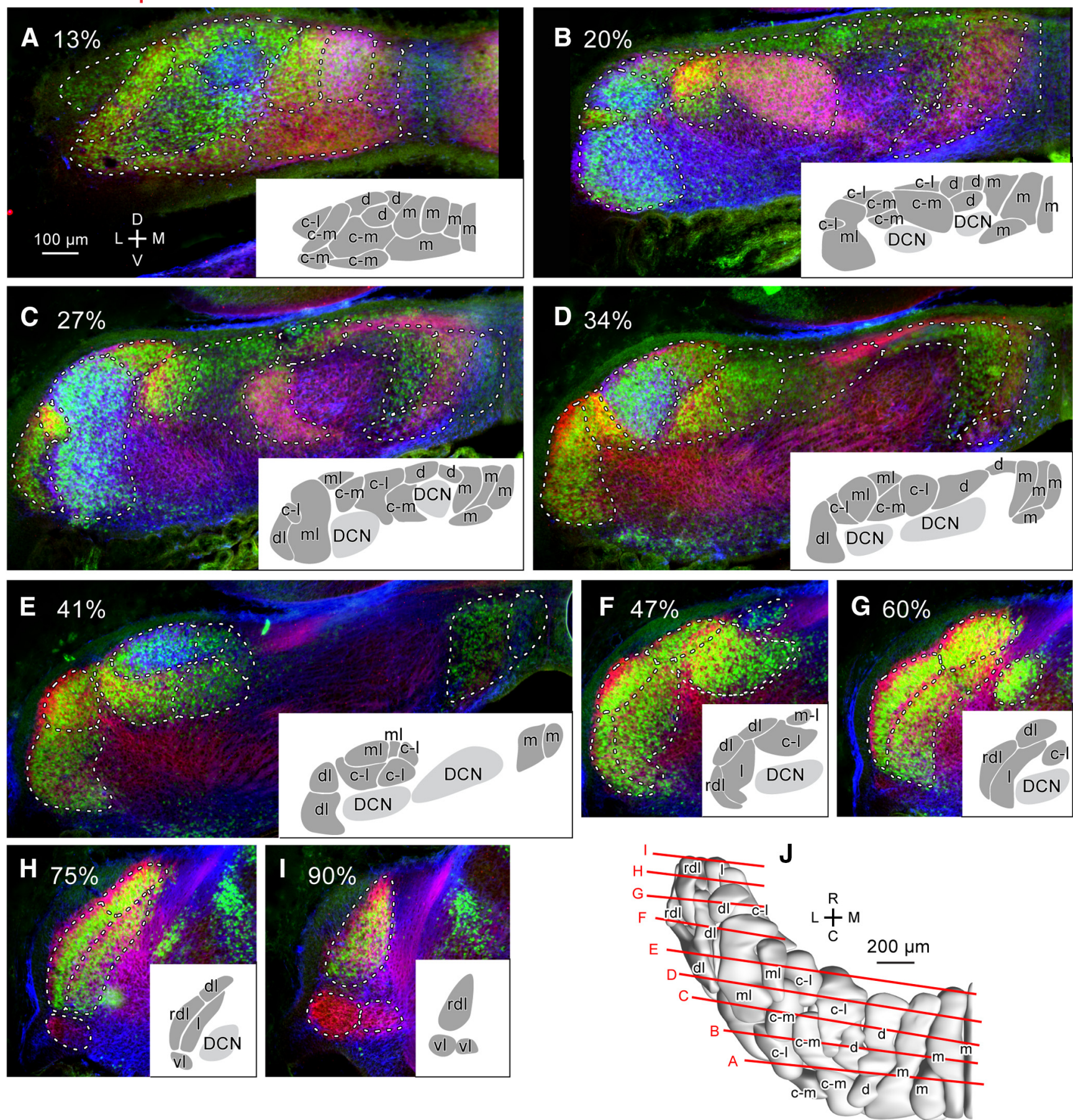

Figure 4. Purkinje cell clusters in coronal sections of the left E16.5 mouse cerebellum identified by marker expression profiles. A-I, Sections at different caudorostral levels indicated by percentile. Fluorescent signals of immunostaining for EphA4 (red), Pcdh10 (blue) and FoxP2 (green) are merged. White dashed lines indicate the boundary of recognized clusters. Inset in each panel shows drawings of recognized clusters at half magnification. Names of each cluster were given later based on the lineage analysis (c.f. Fig. 7). $\boldsymbol{J}$, Dorsal view of the 3D reconstruction of clusters. Red lines indicate the rostrocaudal level of the section in each panel. Scale bar in A $(100 \mu \mathrm{m})$ applies to panels $\mathbf{A}-\mathbf{I}$. Abbreviations, C-I, C-m, d, dl, I, m, ml, rdl, vl, names of the lineage of clusters; C, caudal; D, dorsal; DCN, deep cerebellar nucleus; L, lateral; M, medial; R, rostral.V, ventral. 


\section{E17.5 EphA4FoxP2Pcdh10}
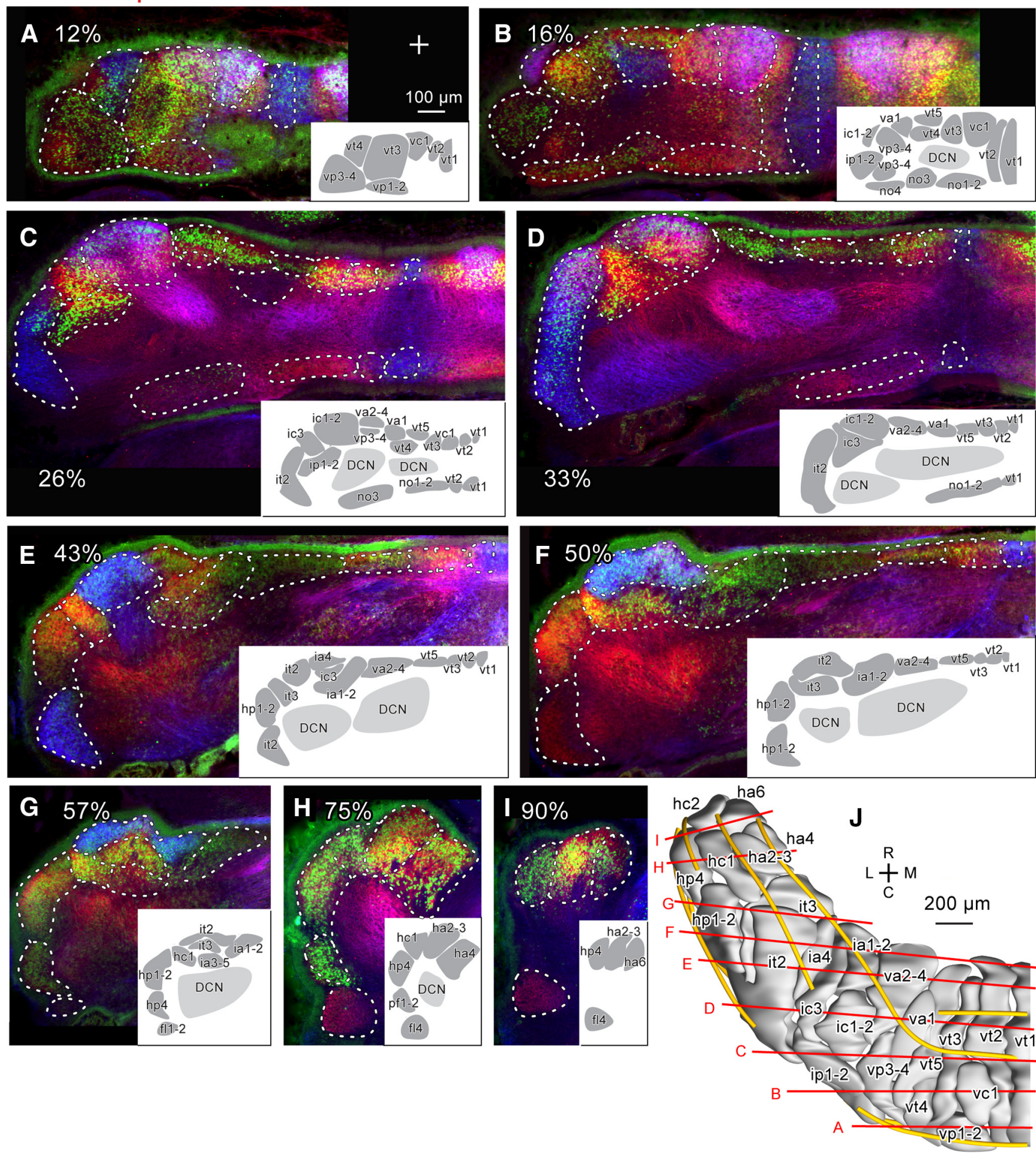

Figure 5. Purkinje cell clusters in coronal sections of the left E17.5 mouse cerebellum identified by marker expression profiles. A-I, Sections at different caudorostral levels indicated by percentile. Fluorescent signals of immunostaining for EphA4 (red), Pcdh10 (blue) and FoxP2 (green) are merged. White dashed lines indicate the boundary of recognized clusters. Inset in each panel shows drawings of recognized clusters at half magnification. Names of each cluster were basically adopted from Fujita et al. (2012), but see Results. $\boldsymbol{J}$, Dorsal view of the 3D reconstruction of clusters. Yellow lines indicate immature fissures. Red lines indicate the rostrocaudal level of the section in each panel. Scale bar in $\boldsymbol{A}(100 \mu \mathrm{m})$ applies to panels $\boldsymbol{A}-\boldsymbol{I}$. Abbreviations, fl1-2, fl4, ha2-3, ha4, ha6, hc1, hc2, hp1-2, hp4, ia1-2, ia3-5, ia4, ic1-2, ic3, ip1-2, it2, it3, no1-2, no3, no4, pf1-2, va1, va2-4, vc1, vp1-2, vp3-4, vt1, vt2, vt3, vt4, vt5, names of E17.5 clusters; C, caudal; D, dorsal; DCN, deep cerebellar nucleus; L, lateral; M, medial; R, rostral.V, ventral. 
E14.5 TM10.5 G2A::Ai9
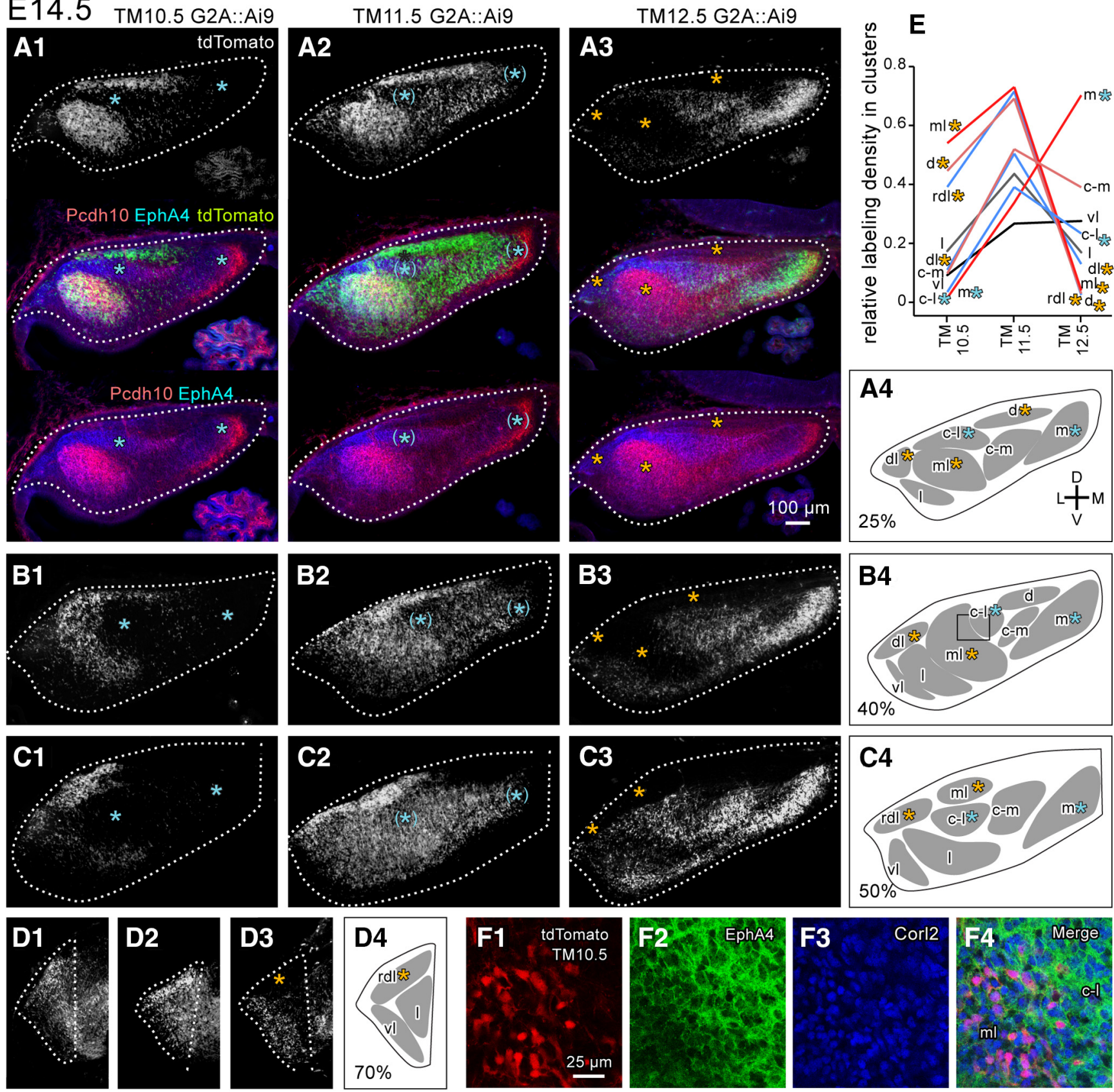

Figure 6. Spatial distribution of E10.5-, E11.5-, and E12.5-born PCs in the E14.5 cerebellum. $\boldsymbol{A}-\boldsymbol{D}$, Images of coronal sections of the left cerebellum of G2A::Ai9 embryos which had tamoxifen injection at E10.5 (subpanel 1), E11.5 (subpanel 2) and E12.5 (subpanel 3) at different rostrocaudal levels. The top, center and bottom panels show images of the tdTomato fluorescence signal (top), three signals merged, and signals of tdTomato fluorescence (green) and immunostaining signals of Pcd10 (red) and EphA4 (blue) in $(\boldsymbol{A})$, while only the tdTomato fluorescence signal is shown in $\boldsymbol{B}-\boldsymbol{D}$. The tdTomato signal indicates neurons (mostly PCs) that expressed Neurog2-CreER at the time of tamoxifen injection. White dashed lines indicate the coutour of the cerebellum. Subpanel 4 shows schematic drawings of identified clusters. Blue and Orange asterisks indicate (position of) the E10.5-PC-sparse and E12.5PC-sparse clusters, respectively. $\boldsymbol{E}$, Relative labeling density in nine E14.5 clusters. The plotted data were the average of the tdTomato fluorescence signal in the digital file (0-255) measured in 9-18 square areas of $900 \mu \mathrm{m}^{2}$ located inside the identified cluster in 3-6 sections. The same brightness and contrast adjustment was done in all sections. $\boldsymbol{F}$, Confocal high-magnification image of immunostaining and tdTomato expression at the junction between the $\mathrm{ml}$ and c-I clusters in the AM10.5 G2A::Ai9 embryo, showing expression of tdTomato (E10.5-born neurons) colocalized exactly with Corl2 (PC marker) at the cellular level in the ml cluster. The area of this image is indicated in B4 with a square. Abbreviations, c-I, c-m, d, dl, I, m, ml, rdl, vl, names of E14.5 clusters; C, caudal; D, dorsal; L, lateral; M, medial; R, rostral.V, ventral. 
Table 2: Nine E14.5 PC clusters, their molecular expression profiles, PC birthdates and fates at E17.5

\begin{tabular}{|c|c|c|c|c|c|c|c|c|c|c|}
\hline E14.5 PC cluster & & $\mathrm{m}$ & $\mathrm{d}$ & $\mathrm{c}-\mathrm{m}$ & $c-1$ & $\mathrm{ml}$ & $\mathrm{dl}$ & rdl & I & $\mathrm{vl}$ \\
\hline Molecular & Corl2 & $+\sim+++$ & ++ & +++ & ++ & + & +++ & +++ & ++ & ++ \\
\hline expression & Pcdh10 & $-\sim++$ & ++ & $+\sim+++$ & $+/-$ & +++ & $+/-$ & ++ & + & $+/-$ \\
\hline profile & EphA4 & $-\sim++$ & - & ++ & ++ & +++ & ++ & +++ & ++ & $+/-$ \\
\hline & FoxP2 & $+\sim+++$ & +++ & + & ++ & +++ & ++ & +++ & ++ & - \\
\hline PC birthdate & & $\begin{array}{l}- \\
\text { E11.5, } \\
\text { E12.5 }\end{array}$ & $\begin{array}{l}\text { E10.5, } \\
\text { E11.5, } \\
-\end{array}$ & $\begin{array}{l}\text { E10.5, } \\
\text { E11.5, } \\
\text { E12.5 }\end{array}$ & $\begin{array}{l}- \\
\text { E11.5, } \\
\text { E12.5 }\end{array}$ & $\begin{array}{l}\text { E10.5, } \\
\text { E11.5 } \\
-\end{array}$ & $\begin{array}{l}\text { E10.5, } \\
\text { E11.5 } \\
-\end{array}$ & $\begin{array}{l}\text { E10.5, } \\
\text { E11.5 } \\
-\end{array}$ & $\begin{array}{l}\text { E10.5, } \\
\text { E11.5, } \\
\text { E12.5 }\end{array}$ & $\begin{array}{l}\text { E10.5, } \\
\text { E11.5, } \\
\text { E12.5 }\end{array}$ \\
\hline $\begin{array}{l}\text { Fate at E17.5 } \\
\qquad(\text { E17.5 cluster })\end{array}$ & & $\begin{array}{l}\text { vt1, vt2, vc1, } \\
\text { vp1-2, vt3, } \\
\text { no1-2 }\end{array}$ & $\begin{array}{l}\text { vt4, vt5, } \\
\text { va1 }\end{array}$ & $\begin{array}{c}\text { vp3-4, ic1-2, } \\
\text { no4, no5, } \\
\text { no3, ic3 }\end{array}$ & $\begin{array}{c}\text { va2-4, ip1-2, } \\
\text { ia1-2, it3, } \\
\text { ha4, ia3-5 }\end{array}$ & it2, ia4 & $\begin{array}{l}\text { hp1-2, hc1, } \\
\text { ha1, ha2-3 }\end{array}$ & $\begin{array}{l}\text { hp3, hp4, } \\
\text { pf }\end{array}$ & $\begin{array}{l}\text { ha5, ha6, } \\
\text { hc2 }\end{array}$ & $\begin{array}{r}\mathrm{fl} 1-2, \mathrm{fl} 3 \\
\mathrm{fl} 4, \mathrm{fl} 5\end{array}$ \\
\hline
\end{tabular}

In sum, distribution patterns of birthdate-specific PCs were tightly linked with the PC clusters at E14.5. Consequently, the birthdate-specific labeling of PCs were expected to be a useful tool to track the lineage of the 14.5 PC clusters.

\section{Tracking birthdate-specific subsets of PCs from E14.5 to E17.5}

Since the $\mathrm{m}$ and c-I clusters were E10.5-PC-sparse and $\mathrm{d}, \mathrm{ml}, \mathrm{dl}$ and rdl clusters were E12.5-PC-sparse at E14.5 (preceding section), we considered that lineages of these clusters were also E10.5-PC-sparse or E12.5-PC-sparse in later developmental stages. Therefore, we examined distributions of tdTomato-labeled PCs in PC clusters that were identified by immunostaining of Pcdh10 and EphA4 and referring to our preceding analysis of clusters (Figs. 2-5) in serial coronal sections of TM10.5 and TM12.5 G2A::Ai9 cerebellums at E14.5, E15.5, E16.5 and E17.5 (Fig. 7). E10.5-PC-sparse clusters and E12.5-PC-sparse clusters were identified among all clusters distinguished at each stage (blue and orange areas in panels of Fig. $7 A-D$ ). This observation indicated that medially- and centrolaterally-located E10.5-PC-sparse clusters belonged to the lineage of the $\mathrm{m}$ and $\mathrm{c}-\mathrm{I}$ clusters (designated as $\mathrm{m}$ and $C-1$ lineage clusters), respectively, at E15.5-E17.5 (Fig. $7 B-D$, blue). Similarly, it was indicated that medially-located E12.5-PC-sparse clusters were d lineage clusters, whereas laterally-located E12.5-PC-sparse clusters were either the $\mathrm{ml}$, dl or rdl lineage clusters, respectively (Fig. $7 B-D$, orange). Finally, it was indicated that medially, laterally, and ventrolaterally-located clusters that contained both E10.5-born and E12.5-born PCs were c-m, I, and vl lineage clusters (Fig. 7B-D, neither blue or orange), respectively.

The above conclusion was supported by the consistency of the marker expression profile of lineage clusters. The $\mathrm{m}, \mathrm{d}, \mathrm{c}-\mathrm{m}, \mathrm{c}-\mathrm{I}, \mathrm{l}, \mathrm{vl}$ lineage clusters showed similar marker expression profiles to those of their original E14.5 clusters, although minor changes in the expression profile were sometimes recognized in some cases (Table 4). Among $\mathrm{ml}$, dl, and rdl lineage clusters, which were E12.5PC-sparse and located next to one another in the lateral cerebellum, the ml cluster at E14.5 and its daughters, or the $\mathrm{ml}$ lineage clusters, at later stages consistently showed strong Pcdh10 expression and were identified consequently (lateral orange in Fig. 7A2, B2, C2, D2). The $\mathrm{dl}$ and $\mathrm{rdl}$ lineage clusters were distinguished based on denser E10.5-born PCs and Pcdh10 expression and more rostrolateral positioning of rdl lineage clusters than dl lineage clusters (Table 4). Since their distinction was not necessarily very clear, we sometimes indicate them together by "dl+rdl" in this report.

As a whole, the birthdate-specific labeling of PCs allowed us to identify the lineage of all PC clusters between E14.5 and E17.5 (Table 3).

At E17.5, the $m$ lineage clusters were located in the most medial area (blue, vt1, vt2, vt3, vc1, vp1-2, in Fig. $7 D 4$ ), whereas the $d$ lineage clusters were located in the laterally neighboring area (orange, va1, vt5, vt4 in Fig. 7D4). The location of other lineages of clusters was more complicated. All c-m lineage clusters and some c-I lineage clusters were intermingled and located in the intermediate position lateral to the d lineage clusters (grey, vp3-4, ic1-2, ic3 and light blue, ia1-2, va2-4, ip1-2, in Fig. 7D4). More lateral were all $\mathrm{ml}$ lineage clusters and some c-I lineage clusters (yellow, it2, ia4 and light blue, it3 in Fig. 7D4). All $\mathrm{dl}+\mathrm{rdl}$ and I lineage clusters and some c-I linage clusters were located in the most lateral position (yellow, hp1-2, hc1, ha2-3, hp4, grey, hc2, ha6, and light blue, ha4 in Fig. 7D4). All vl lineage clusters were located in the ventrolateral edge of the E17.5 cerebellum (not shown in Fig. 7D4).

The results demonstrated that the positional relationship among clusters changed in some places during the period between E14.5 and E17.5, indicating that the separate migration of divided clusters is one of the bases for the rearrangement of the compartmental organization of the cerebellum. Furthermore, the marker expression profiles of separated clusters that belonged to the same lineage changed differently to some extent in some cases (Table 4).

\section{Spatial differentiation of the c-I lineage clusters during the period from E14.5 to E17.5}

Among the 37 identified clusters in the E17.5 cerebellum, c-l lineage clusters were located in the most widelyseparated areas (light blue, ip1-2, ia1-2, va2-4, it3, ia3-5 and ha4 in Fig. 7D4). Although originating from a single c-I cluster, the c-l lineage clusters were located separately at various rostrocaudal and mediolateral positions in the E17.5 cerebellum (Fig. 8G). As described in the preceding section, all the c-I lineage clusters were E10.5-PC-sparse, which facilitated their identification, and showed no expression of Pcdh10 (scarce red or green signals in 
TM10.5 E14.5
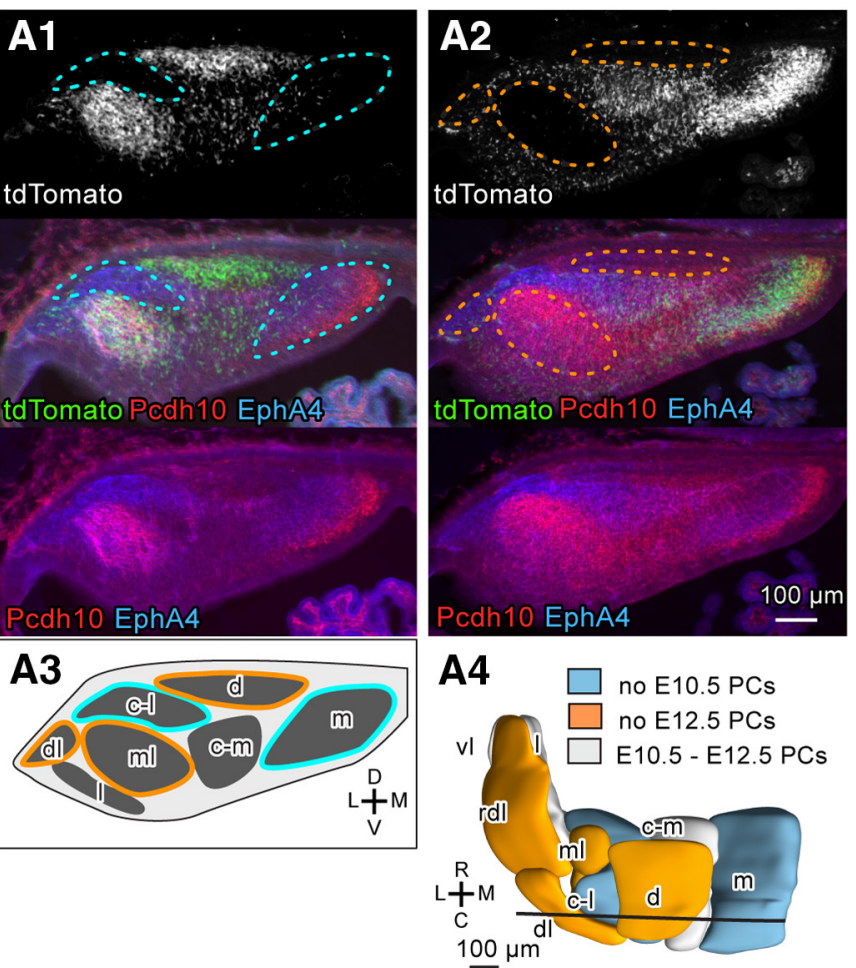
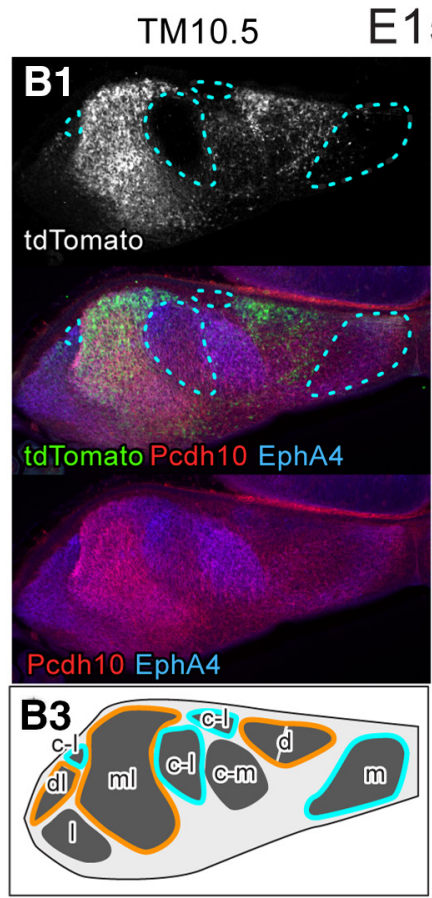
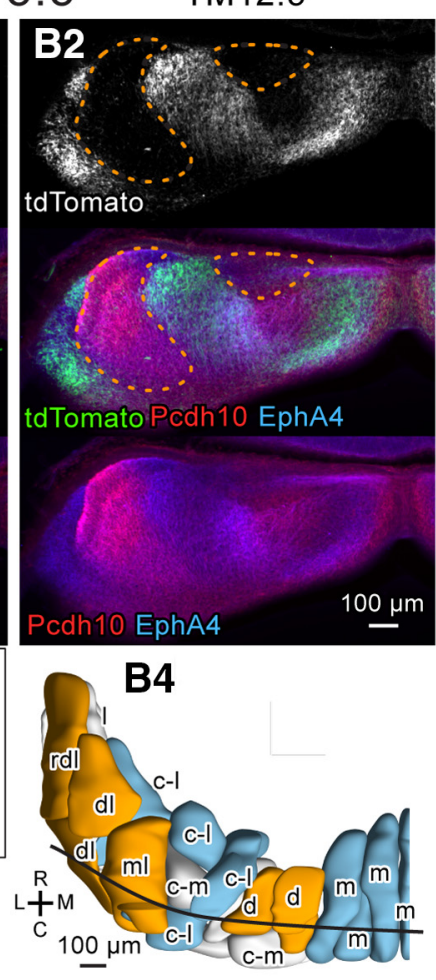

TM10.5 E17.5

TM12.5
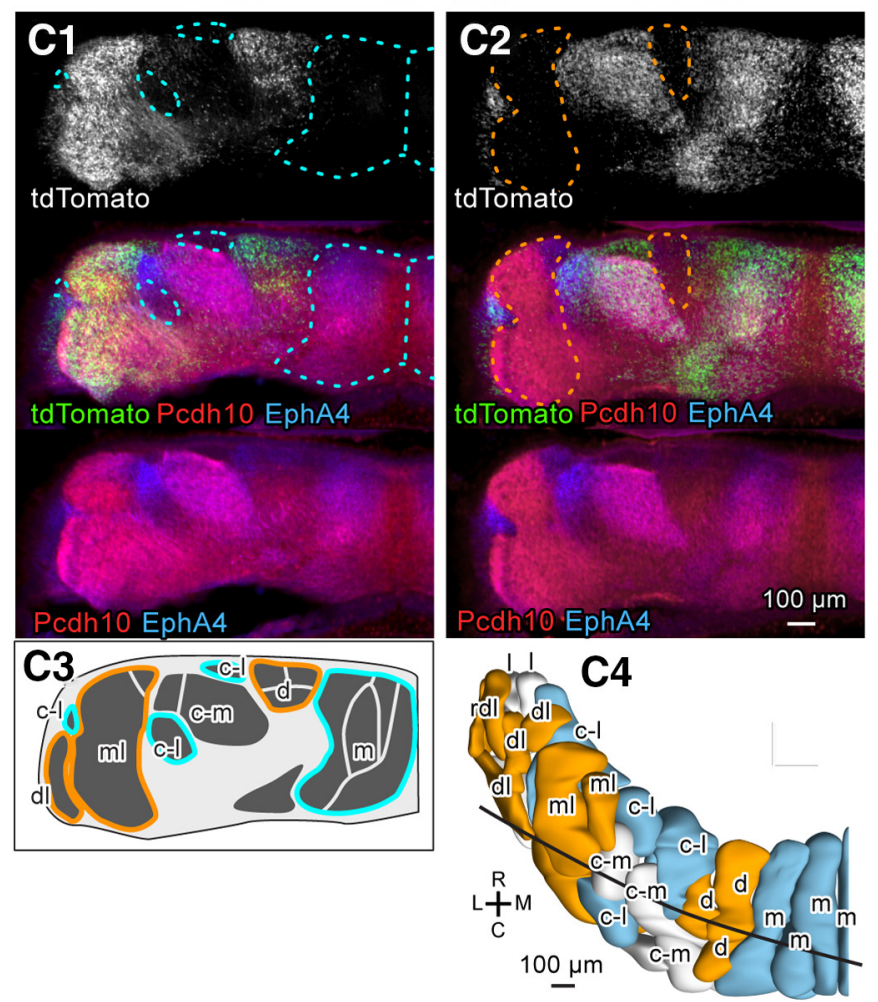
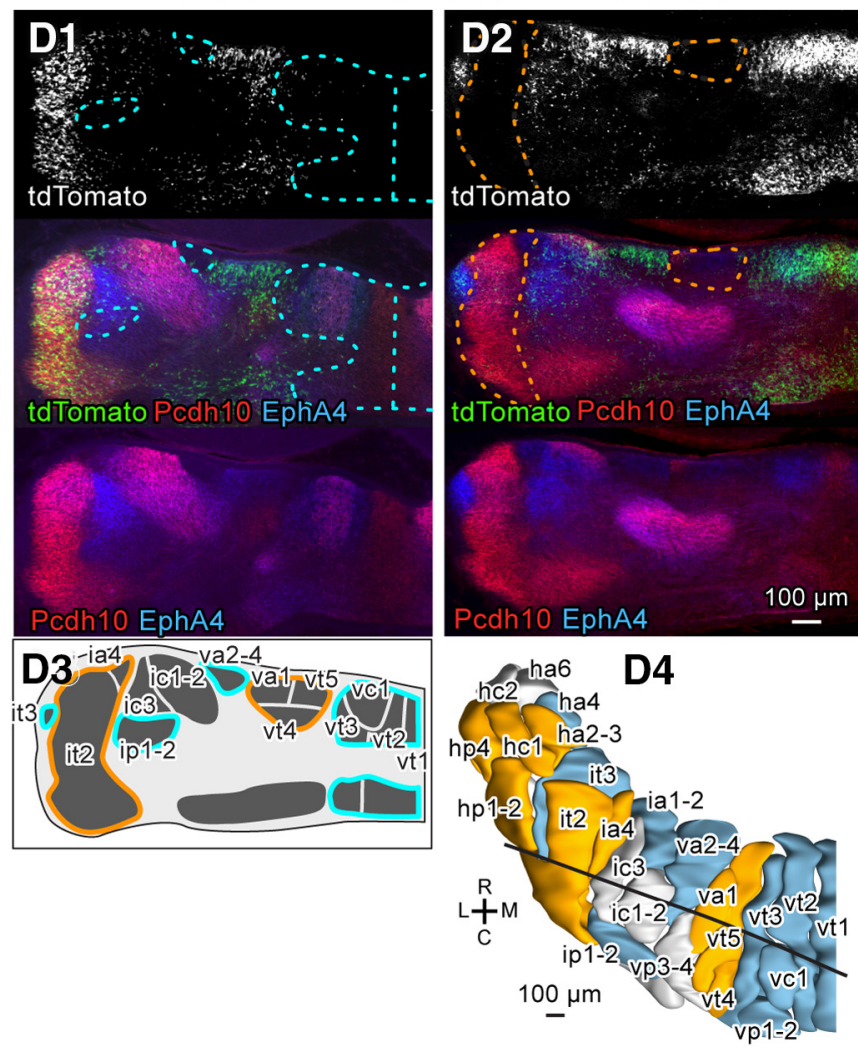

Figure 7. Identification of E10.5-PC-sparse and E12.5-PC-sparse clusters in the E14.5-E17.5 cerebellums. $\boldsymbol{A}-\boldsymbol{D}$, Images of coronal sections of the left TM10.5 and TM12.5 cerebellums at nearly the same level (subpanel 1 and 2, respectively) and schematic drawing of clusters (subpanel 3) and dorsal view of the 3D scheme (subpanel 4) of the E14.5 (A), E15.5 (B), E16.5 (C), and E17.5 (D) cerebellums. In subpanels 1 and 2, the tdTomato fluorescence signal (top), three signals merged, and signals of tdTomato fluorescence 
continued

(green) and immunostaining signals of Pcd10 (red) and EphA4 (blue) are shown from the top to the bottom. Blue and orange colors in dashed lines (in subpanels in 1 and 2), circumscribing lines (in subpanels 3), and clusters in the 3D schemes (in subpanels 4) indicate E10.5-PC-sparse and E12.5-PC-sparse clusters. Black line in the 3D scheme indicates the position of the coronal section in subpanels 1-3. Abbreviations, c-I, c-m, d, dl, I, m, ml, rdl, vl, names of E14.5 clusters; ha2-3, ha4, ha6, hc1, hc2, hp1-2, hp4, ia1-2, ia4, ic1-2, ic3, ip1-2, it2, it3, va1, va2-4, vc1, vp1-2, vp3-4, vt1, vt2, vt3, vt4, vt5, names of E17.5 clusters; C, caudal; D, dorsal; L, lateral; M, medial; R, rostral.V, ventral.

circumscribed clusters in Fig. $8 A-F)$ and various expression of EphA4 (blue signals in some circumscribed clusters in Fig. 8A-F).

To further clarify the spatial differentiation of c-I lineage clusters during the period between E14.5 and E17.5, we examined positional relationships between c-I lineage clusters and neighboring clusters (Fig. 9). At E14.5, the c-I cluster was located lateral to the c-m cluster and medial to the ml cluster: again, the c-l cluster was identified by the weak Pcdh10 expression, weak Corl2 expression and the lack of E10.5-born PCs (Fig. 9A1, Table 2). It occupied the mid-lateral part of the center of the cerebellum with its dorsocaudal part extended laterally to the superficial area dorsal to the ml cluster (Fig. 9A1, B1). The rostral part of the $\mathrm{C}$-I cluster was more extended laterally in the position rostroventral to the $\mathrm{ml}$ cluster (arrowheads in Fig. 9A1). The rostromedial part of the c-l cluster had a slightly stronger Corl2 expression than the rest of the c-l cluster (Fig. 9A1, asterisk) and adjoined medially with the c-m cluster which had strong Pcdh10 expression (Fig. 9C1,D1).

Table 3: Definition of E17.5 PC clusters

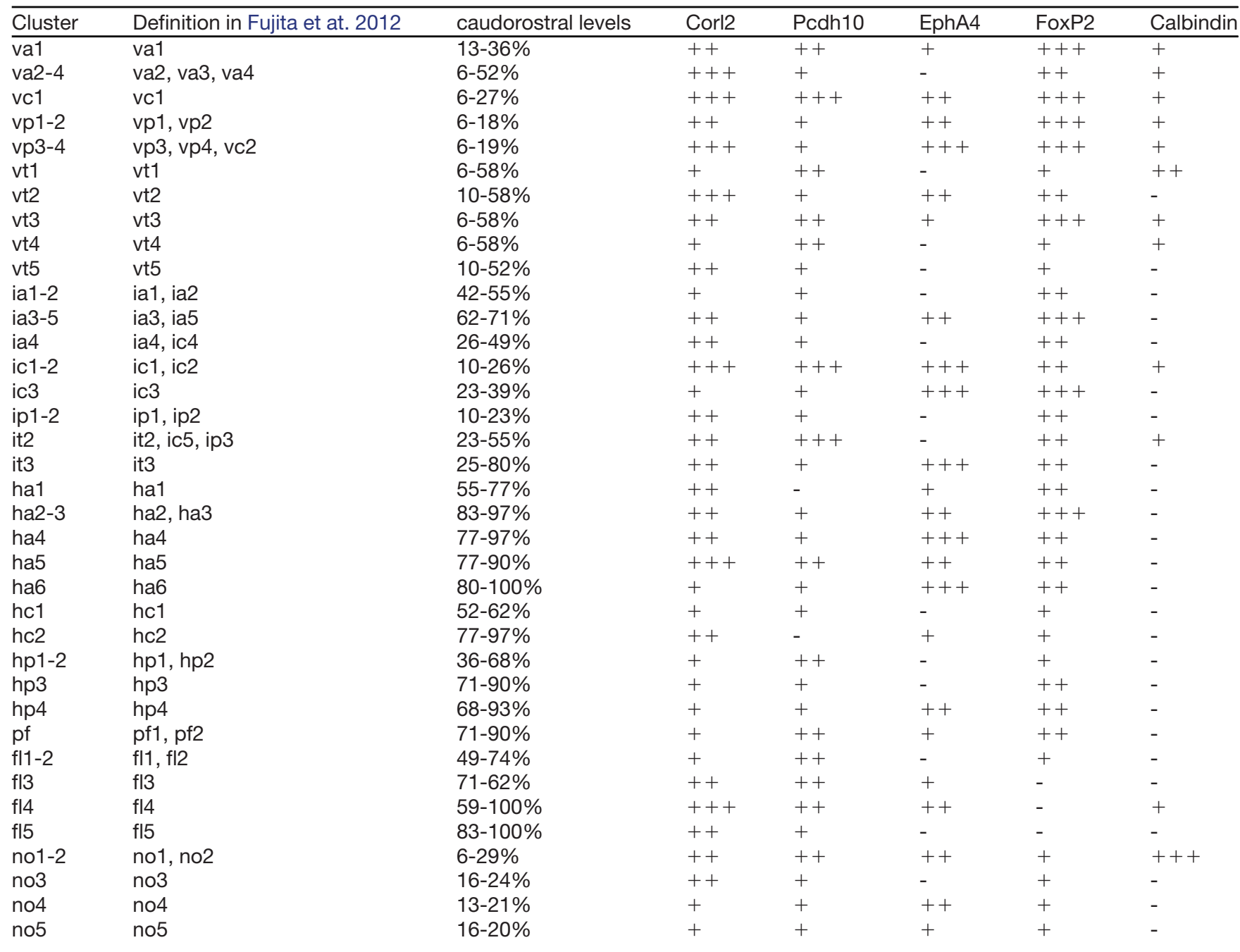

Correspondence to the definition in Fujita et al. (2012), and location of the cluster within the caudorostral level of the cerebellum are shown (columns 2,3). Relative intensity in immunostaining in the present study (columns 4-8) was generally consistent with the previous result (Fujita et al., 2012), but showed some minor differences. In cluster names, "v", "i", "h", "a", "p", "t", "c", "pf", "fl", "no" means vermal, intermediate (paravermis), hemisphere, anterior, posterior, translobular (anterior+ posterior), central, parafloccular, floccular, nodular, respectively. The numeral (such as "1" in "vp1-2") counts the cluster from the medial to the lateral side in each category ("it1" is absent). 
Table 4: Changes in the molecular expression profile in PC clusters from E14.5 to E17.5

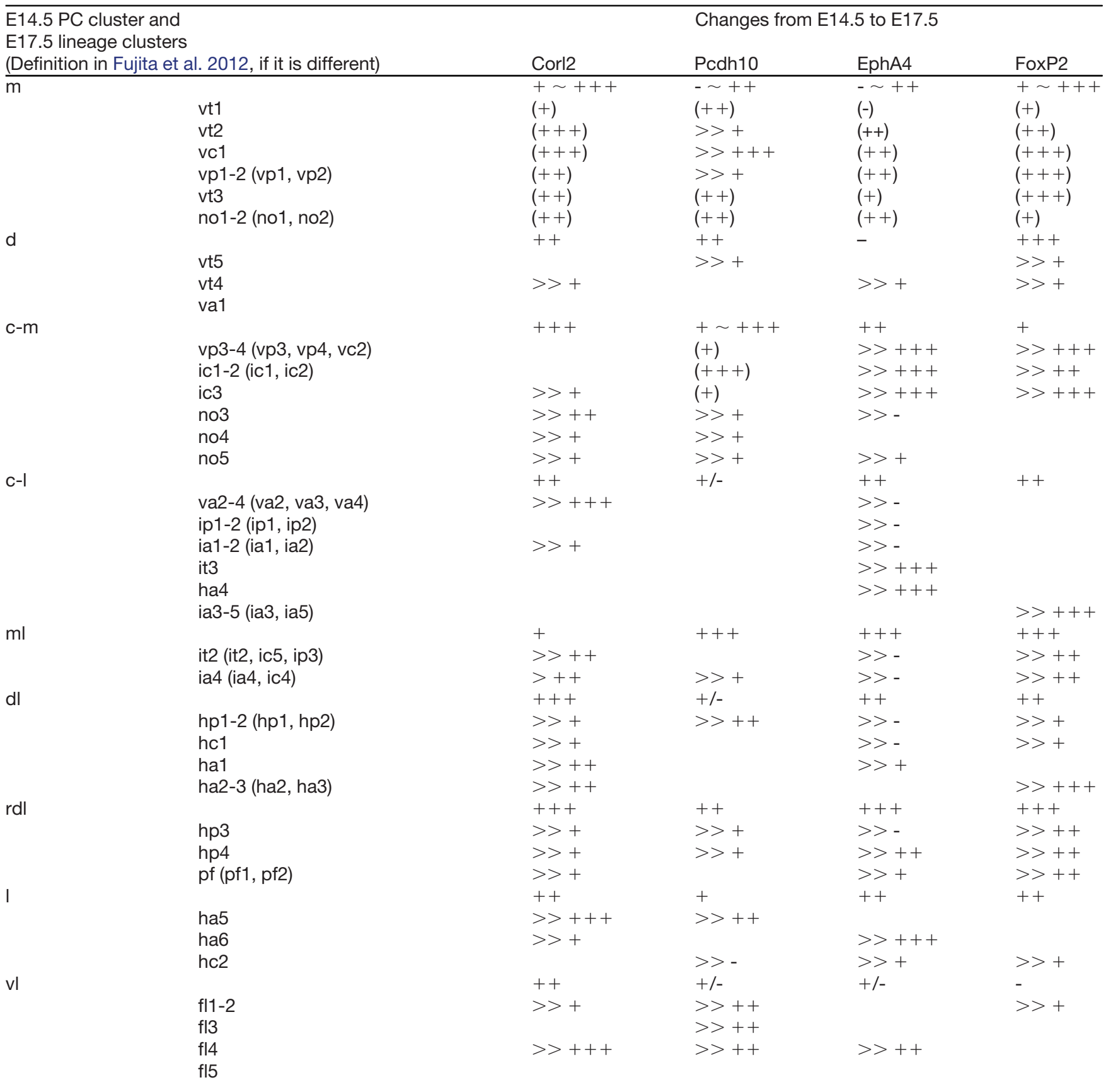

“ " indicates the gradient in the molecular expression intensity.

" $>>$ " indicates the change in the molecular expression intensity

At E15.5, three major rearrangements occurred in the cI cluster. Firstly, the most medial part of the c-I lineage cluster was recognized as a separate daughter cluster because of its strong Corl2 expression (single asterisk in Fig. $9 A 2, B 2, C 2, D 2)$. Secondly, the rostrolateral part of the cI lineage cluster migrated further laterally in areas rostral and ventral to the $\mathrm{ml}$ lineage cluster and increased in EphA4 expression (arrowheads in Fig. 9A2). The caudolateral part of the c-I lineage cluster retracted medially. Lastly, the lateral part of the c-m lineage cluster, (Fig. $9 A 2$, B2, double asterisk), migrated laterally at the position ventral to the c-l lineage cluster to separate the ventral portion of the $\mathrm{c}-\mathrm{I}$ lineage cluster into the rostral and caudal parts (Fig. 9C2, D2).

At E16.5, the separation of the c-I cluster that started at E15.5 became clearer. The most lateral part of the c-m lineage cluster further migrated laterally to separate the rostral and caudal parts of the c-l lineage cluster completely (Fig. 9A3, B3, C3, D3, double asterisk). This lateral migration of the $\mathrm{c}-\mathrm{m}$ lineage cluster confirmed our previous observation with pcdh10 reporter mice (Vibulyaseck et al., 2017). The rostral part of the c-I lineage cluster was 
E17.5 TM10.5
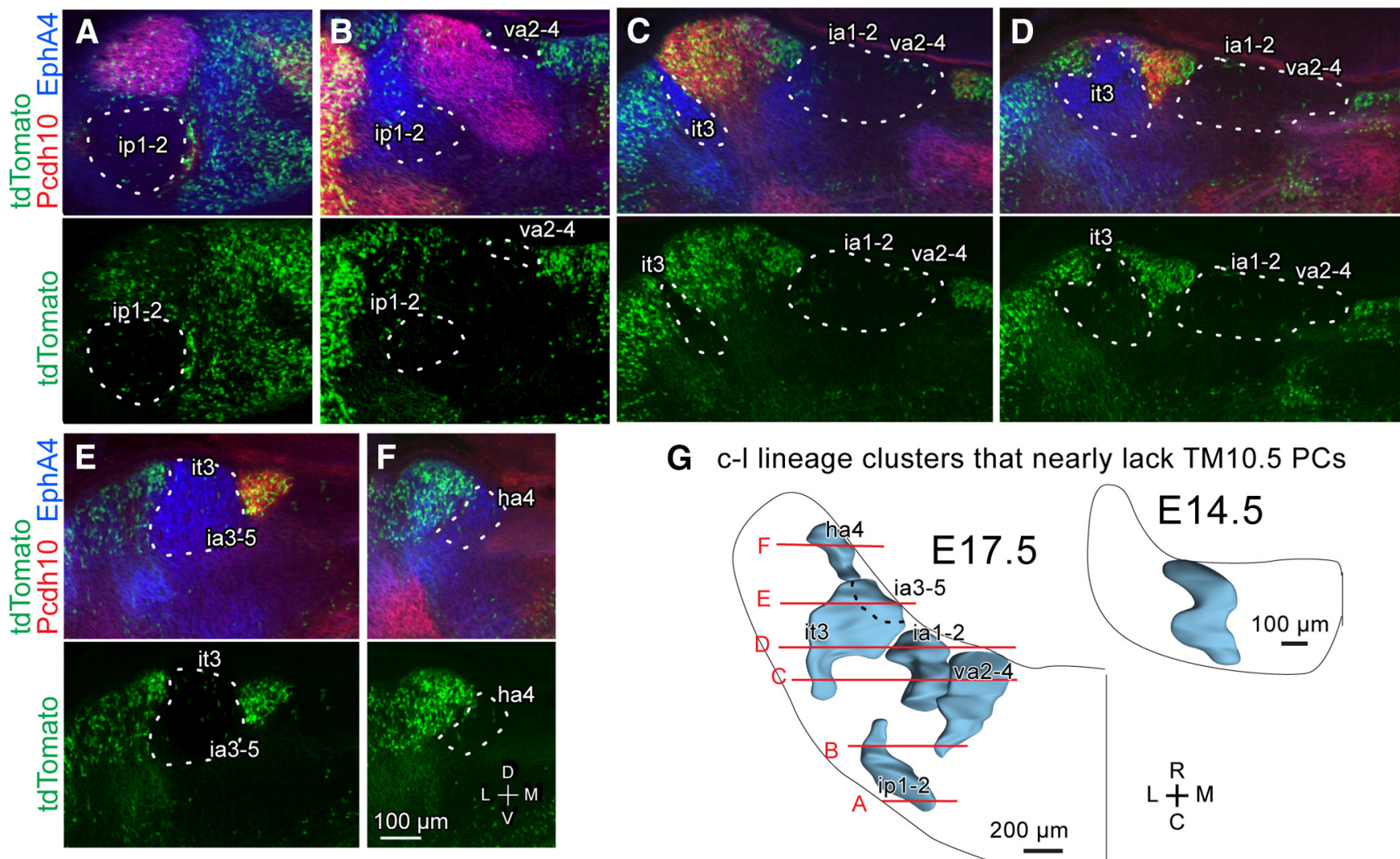

G $\quad$-l lineage clusters that nearly lack TM10.5 PCs

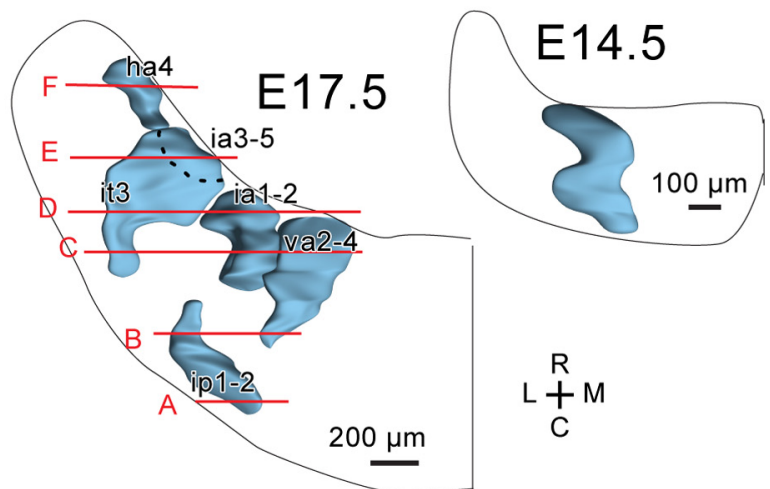

Figure 8. Separated distribution of the E10.5-PC-sparse c-I lineage clusters in the E17.5 cerebellum. $\boldsymbol{A}-\boldsymbol{F}$, Images of a part of the coronal section at various rostrocaudal levels of the left cerebellum. The top subpanel shows the merged signal of Pcdh10 immunostaining (red), EphA4 immunostaining (blue) and tdTomato expression indicating E10.5-born PCs (green), whereas the bottom subpanel shows only tdTomato expression. White dashed lines demarcate E10.5-PC-sparse areas. Scale bar in $\boldsymbol{F}$ applies $\boldsymbol{A}-\boldsymbol{F}$. $\boldsymbol{G}$, Dorsal view of the 3D scheme of the c-I lineage clusters in the left E17.5 cerebellum, compared with the single c-I cluster in the left E14.5 cerebellum. Black dashed line indicate the contour of the ia3-5 cluster which is located ventral to the it3 cluster. Abbreviations, ha4, ia1-2, ia3-5, it3, ip1-2, va2-4, names of E17.5 clusters; C, caudal; D, dorsal; L, lateral; M, medial; R, rostral.V, ventral.

spread widely in the mediolateral direction (arrowheads in Fig. 9A3, B3) and subdivided into three daughter subclusters that were recognized by different molecular expression profiles (Fig. 9A3, B3). The lateral daughter subcluster migrated laterally and also elongated caudally (blue in Fig 9B3).

At E17.5, the separated c-I lineage clusters (ip1-2, ha4, it3, ia1-2, and va2-4 clusters in Fig. 9A4, B4) spread in the mediolateral direction at different rostrocaudal levels (arrowheads in Fig. 9A4). The laterally migrating Pcdh10positive c-m cluster (ic1-2 cluster) faced the dorsal surface of the cerebellum (Fig. 9C4, D4), as reported previously (Vibulyaseck et al., 2017). The appearance of clear PC-free gaps further firmly separated neighboring daughter clusters (e.g. ia1-2 vs va2-4, in Fig. 9A4). Within these daughter clusters, we noticed a developmental change in EphA4 and Corl2 expression; Corl2 expression became much stronger in the va2-4 cluster than other clusters, while EphA4 expression became stronger in the it 3 and ha4 clusters than in the va2-4 and ia1-2 clusters. However, Pcdh10 expression remained weak in these c-I lineage daughter clusters throughout the development from E14.5-17.5 (Fig. 9A4).

\section{The lineage of the c-I cluster at adult}

Our previous study (Fujita et al., 2012) has suggested that the E17.5 clusters that were identified as the c-I lineage clusters in the present study (va2-4, ia3-5, ia1-2, it3, ha4, and ip1-2 clusters in the E17.5 cerebellum, Fujita et al., 2012) become zebrin-negative and-lightly-positive stripes in paravermal and hemispheric areas in the anterior and posterior lobules. We tried to confirm the location of c-l lineage stripes directly in the adult cerebellar cortex by using G2A::Ai9::AldocV mice which show tamoxifeninduced birthdate-dependent labeling of neurons with tdTomato as well as labeling of zebrin-positive PCs with Venus fluorescent protein (Fig. 10A). We have reported the dependency on tamoxifen injection timing of the general pattern of PC labeling in adult G2A::Ai9::AldocV mice (Zhang et al., 2020). Here, we mapped E10.5-PCsparse cortical areas by the observation of cerebellar sections of G2A::Ai9::AldocV mice that received tamoxifen at $E 10.5$ ( $n=2$ mice) to identify c-l lineage stripes.

In the adult mouse cerebellar cortex, the striped expression pattern of zebrin (aldolase $\mathrm{C}$ ) is consistent across individuals and has been identified in detail (Fujita et al., 
E14.5

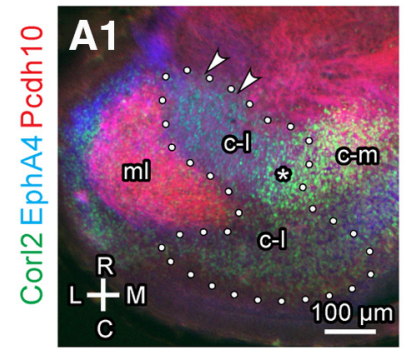

B1
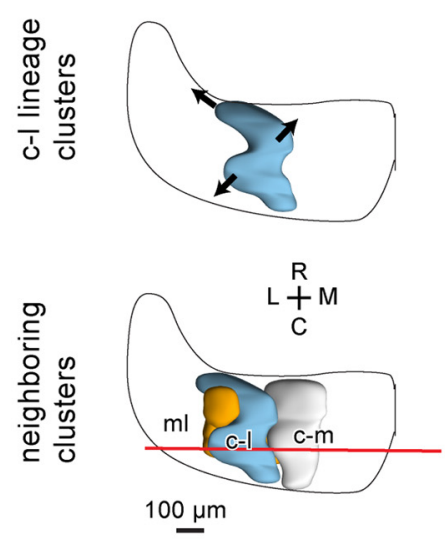

E15.5

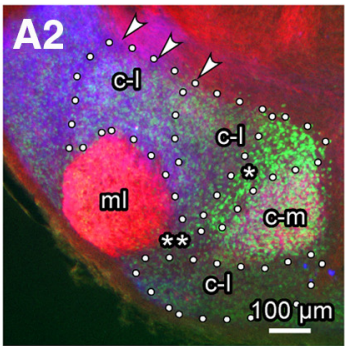

B2

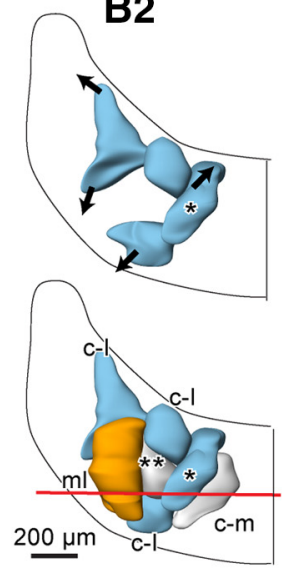

E16.5

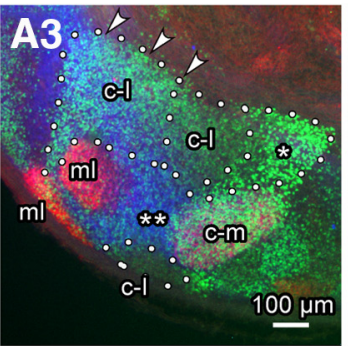

B3
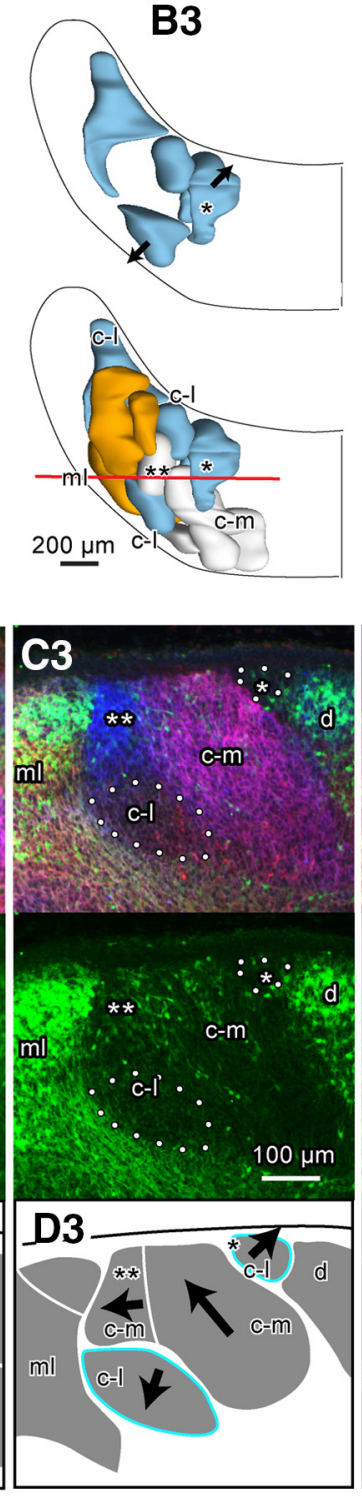

E17.5
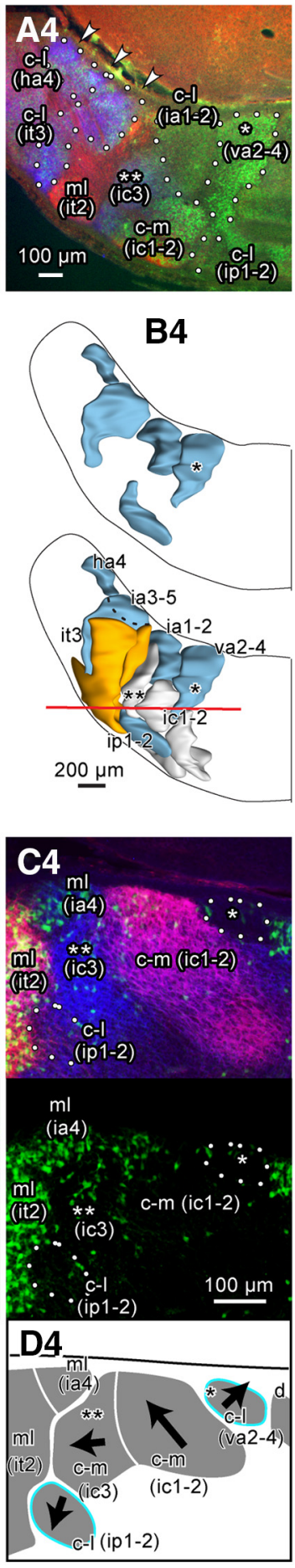
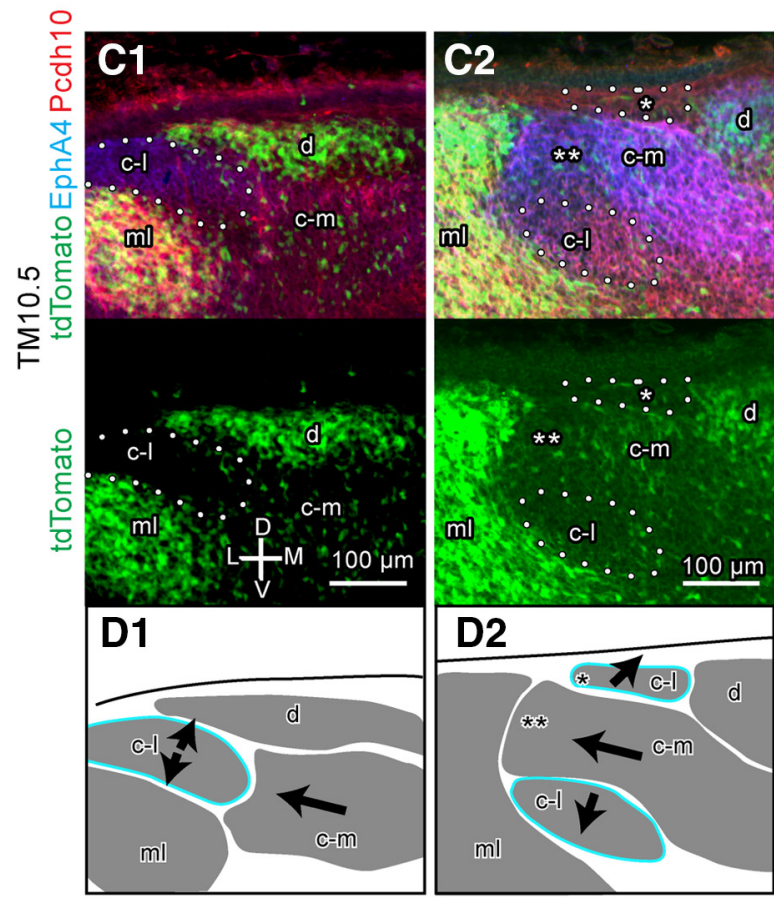

Figure 9. Separation and migration of the c-I lineage cluster from E14.5 to E17.5. A, Horizontal sections of the left paravermal and hemispheric cerebellum at around the central level. Sections were immunostained for Corl2 (green channel), EphA4 (blue channel), and Pcdh10 (red channel). Dashed lines circumscribe c-I lineage clusters. Arrowheads indicate the lateral migration of the rostral c-I lineage clusters. $\boldsymbol{B}$, Dorsal view of the three-dimensional scheme of reconstructed c-l lineage clusters (blue). In the bottom panel, ml and $\mathrm{c}-\mathrm{m}$ lineage clusters (orange and white) are added. Red transversal line indicate the position of the coronal section shown in (B). Black dashed line indicate the contour of the ia3-5 cluster which is located ventral to the it3 cluster in (B4). $\boldsymbol{C}$, Images of a part of the left cerebellum at the junction between the c-I and c-m lineage clusters in coronal sections. The top image shows merged signals of tdTomato expression, which indicates E10.5-born PCs (green) and immunostaining for EphA4 (blue) and Pcdh10 (red). The bottom subpanel shows only the image of only tdTomato labeling. $\boldsymbol{D}$, Schematic drawing of clusters shown in $\boldsymbol{C}$. In $\boldsymbol{A}-\boldsymbol{D}$, columns of subpanels 1-4 are from E14.5, E15.5, E16.5 and E17.5 cerebellums. Dashed lines indicate c-I lineage clusters that are E10.5-PC- 
continued

sparse and weak in Pcdh10 expression in $\boldsymbol{A}$ and $\boldsymbol{C}$. Single asterisks indicate the most medial part of the c-I cluster or the most medial c-l lineage cluster which had higher expression of Corl2 than the rest of the c-l lineage clusters in $\boldsymbol{A}-\boldsymbol{D}$. Double asterisks indicate the most lateral part of the c-m cluster or the most lateral c-m lineage cluster which intercalated the c-I lineage clusters in $\boldsymbol{A}-\boldsymbol{D}$. Arrows indicate the direction of cluster migration. Scale bar in $\mathbf{C 1}(100 \mu \mathrm{m})$ applies to $\mathbf{C 1 - C 4}$. Abbreviations, C-I, C-m, d, ml, names of E14.5 clusters; ha4, ia1-2, ia3-5, ia4, ic1-2, ic3, ip1-2, it2, it3, va2-4, names of E17.5 clusters; C, caudal; D, dorsal; L, lateral; M, medial; R, rostral.V, ventral.

2014; Sarpong et al., 2018). Accordingly, zebrin stripes are described here by using the common nomenclature (Fujita et al., 2014; Sarpong et al., 2018). Zebrin stripes are mostly designated with a numeral or an alphabet character followed by "+" or "--" (Fig. 1C). Note that a pair of stripes in the anterior and posterior lobules, which are named differently, together belongs to the same cerebellar module, because they receive common branching olivocerebellar axons (Sugihara and Shinoda, 2004): for example, a pair of $4+$ stripe in anterior lobules and $5+$ stripe in posterior lobules form the C2 module and thus considered to be the same or linked stripe, which is designated as " $4+/ / 5+$ ".

In lobules I-V, except for stripe $2+$ and the medial part of stripe 2-, most of the paravermal stripes (the lateral part of zebrin stripes 2-, stripes b+, b-, 3+ and 3-) were E10.5-PC-sparse and consequently identified as the fate of $c-I$ lineage clusters (the right circumscribed areas in Fig. 10B1). However, the most lateral part of stripe 3- and the entire stripe 4+ contained a high density of E10.5born PCs. Most of stripe 4- was E10.5-PC-sparse (the left circumscribed area in Fig. 10B1). The most lateral part of stripe 4- and entire stripe $5+$ contained a high density of E10.5-born PCs. Stripe 5- was E10.5-PC-sparse (the left ventral circumscribed area in Fig. 10B1).

In the simple lobule, most of the medial paravermal area had a low-dense distribution of E10.5-born PCs (double asterisks in Fig. 10B1). This area was likely to be derived from c-m lineage clusters, which had similarly a low density of E10.5-born PCs in the embryonic cerebellum (Fig. $7 D$ ). This agrees to the speculation of our previous study that this area originates from E17.5 clusters ic1 and ic2 (Fujita et al., 2012) or cluster ic1-2, which originated from the E14.5 c-m cluster in the present result (Table 2). Zebrin positive stripe $4+$ and medially adjacent zebrin negative area contained a high density of E10.5-born PCs. On the contrary, stripe 4-, except for its lateral part, was E10.5-PC-sparse. Stripe 4- was also E10.5-PCsparse in crus I (the most left circumscribed area in Fig. 10B1). These E10.5-PC-sparse areas were identified as the fate of c-I lineage clusters (circumscribed in Fig. 10B1) occupied a relatively large portion in the paravermal cerebellar cortex in the rostral cerebellum.

Some tdTomato labeling in the molecular layer inside the circumscribed areas indicated the presence of labeled PC dendrite (left magenta labeling in the inset in Fig. 10B1). However, the number of labeled somata that were located within the section (arrowheads in Fig. 10B1-4) was rather small (12 PCs in Panel B1, roughly $1-2 \%$ against the denominator of all PC somata, $\sim 700$, recognized in the background inside the circumscribed areas), supporting our description "mostly lacking" and "E10.5-
PC-sparse". This percentage (1-2\%) was approximately the same as the number of labeled PCs inside the E14.5 $c-I$ cluster (preceding section).

In the rest of cerebellar lobules (lobules VI-X, crus I, crus II, paramedian lobule, copula pyramidis, paraflocculus and flocculus), several areas were E10.5-PC-sparse and identified as the fate of the c-I clusters in the paravermis and hemisphere. These areas included the lateral part of stripe 2- in lobules V-VI (circumscribed in Fig. 10B2), the medial part of stripe 5 - in crus II (circumscribed in Fig. 10B2-B3), and multiple zebrin-negative and faintly zebrin positive stripes in the copula pyramidis and adjacent paramedian lobule (circumscribed in Fig. 10B2, B4). Distribution of E10.5-born PCs in the medial paravermal areas in crus I, crus II and paramedian lobule, which are likely to belong to c-m lineage areas (Fujita et al., 2012; Vibryaseck, 2017), appeared a little denser (double asterisks in Fig. 10B2-4) than the c-I lineage areas. Other stripes in the paravermal and hemispheric areas had a rather dense distribution of E10.5-born PCs.

The E10.5-PC-sparse zebrin stripes that were considered to belong to the c-I lineage were mapped on the unfolded scheme of the entire cerebellar cortex with the zebrin (aldolase C) striped pattern (Fig. 10F; Fujita et al., 2014; Sarpong et al., 2018). The c-l lineage areas were located in zebrin-negative and weakly-positive stripes in the lateral vermis and paravermis in lobules I-V and VIII/ copula pyramidis (red and green in Fig. 10F), and in the medial hemisphere in lobules I-V, simple lobule, crus II and paramedian lobule (blue in Fig. 10F). However, no c-I lineage areas were observed in the central part (lobules VI-VII and apex of crus I) of the cerebellum. Based on the correspondence between the zebrin stripes and cerebellar modules (Sugihara and Shinoda, 2004; Sugihara et al., 2009), the mapped stripes corresponded to the B module (red in Fig. 10F), which projects to the lateral vestibular nucleus (Sugihara et al., 2009; Ruigrok et al., 2015), and most of the C1, C3 and D0 modules, which project to the anterior interposed nucleus (green and blue in Fig. 10F, Table 5). The results demonstrated that the separation and migration of the $\mathrm{c}-\mathrm{I}$ cluster during development forms multiple stripes that mainly correspond to the anterior and posterior parts of the C1/C3 module (Fig. 10C-F), and also the $\mathrm{B}$ module and a part of the D0 module.

\section{Discussion}

The present study demonstrated the spatial development of the compartmental organization of the mouse embryonic cerebellum between E14.5 and E17.5. The lineages of the nine E14.5 PC clusters which transformed into 37 clusters at E17.5 were tracked by birthdate-tagging. Furthermore, it was shown that one of the E14.5 
A Adult
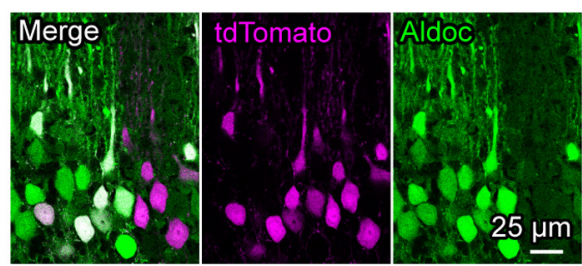

$\mathbf{B}_{\text {Adult }}$
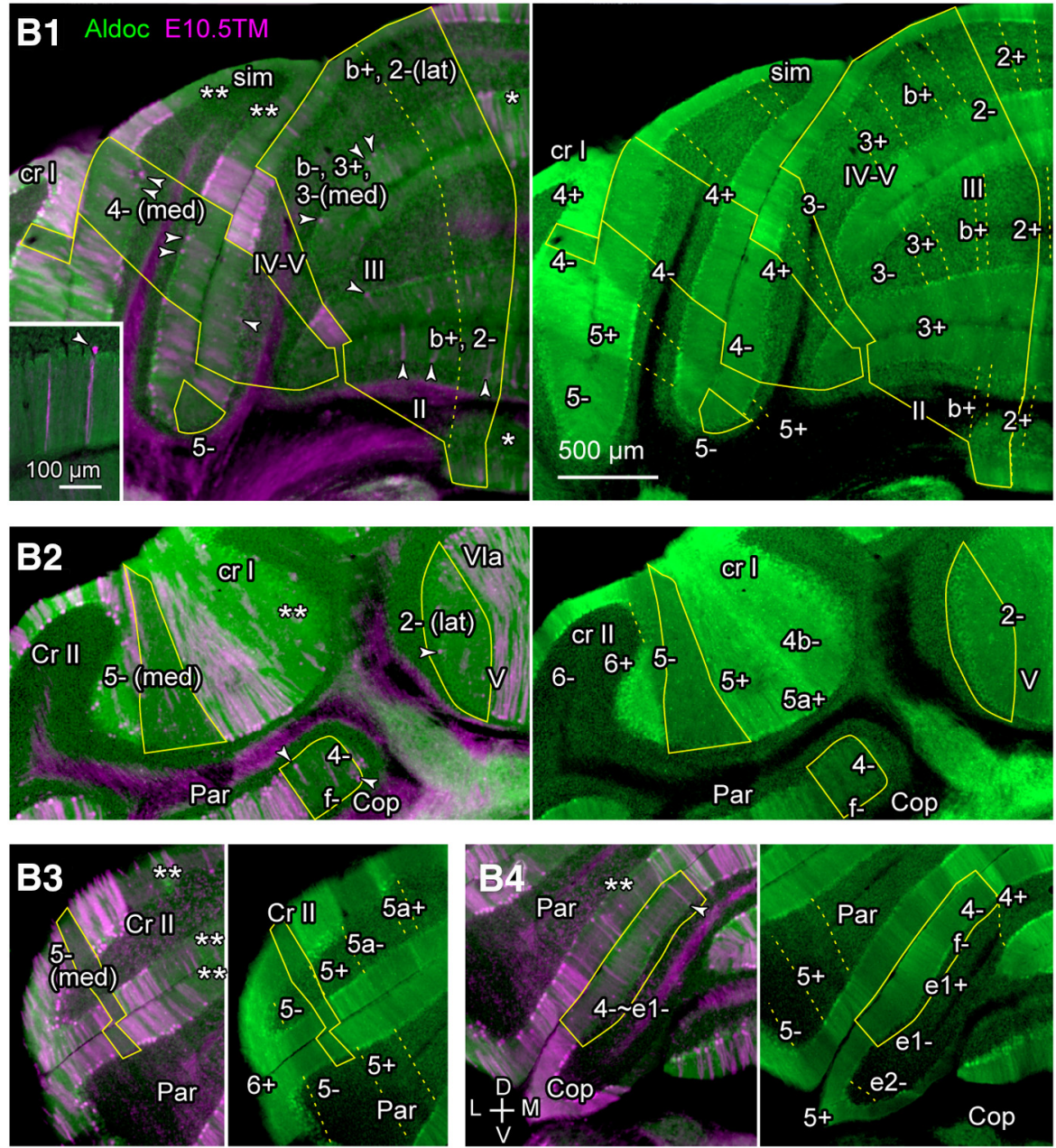

C

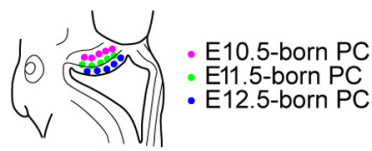

$\mathbf{D}_{\mathrm{E} 14.5}$
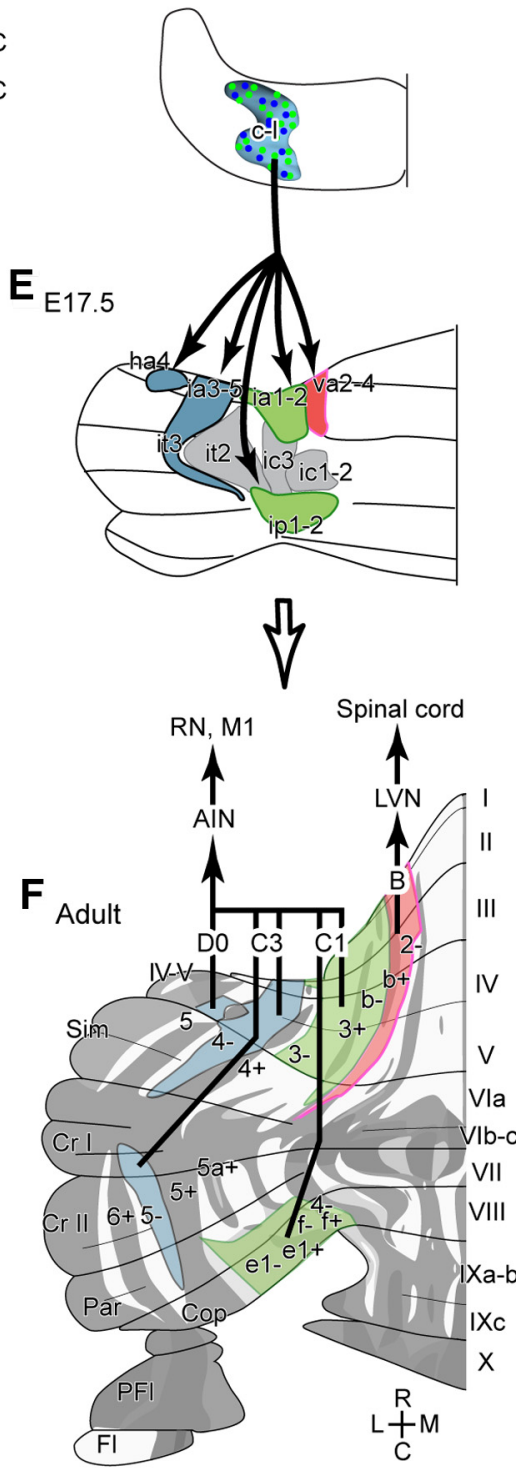

Figure 10. The fate of $\mathrm{c}-\mathrm{I}$ lineage clusters in the compartmentalization of the adult cerebellar cortex. $\boldsymbol{A}$, Confocal high magnification images of the cerebellar cortex of the G2A::Ai9::AldocV mouse in which tamoxifen was given at E11.5, showing tdTomato expression in Purkinje cells. B, Four coronal sections of the left cerebellum at rostral (B1), central (B2) and caudal (B3, B4) levels in the G2A::Ai9::AldocV mouse that received tamoxifen at E10.5 and sacrificed at P42. Superimposed images of magenta (tdTomato representing E10.5-born PCs) and green (Venus representing aldolase C expression) channels are shown in the left, while only green channel (Venus, aldolase C) is shown in the right. E10.5-PC-sparse areas that were considered to belong to the lineage of the c-I cluster were circumscribed by yellow lines. Arrowheads indicated labeled PC somata that were observed within the circumscribed area. Zebrin stripes were indicated in the right panels. Scale bar $(500 \mu \mathrm{m})$ applies to B1-B4. Inset in panel B1 shows sparse Purkinje cell labeling at the dendrite (left) and at the soma and dendrite (arrowhead) in the c-I lineage area. $\boldsymbol{C}$, Schematic of intermittent PC generation in the ventricular zone of the left embryonic cerebellum. $\boldsymbol{D}$, Schematic of the c-l cluster composed of randomly located E11.5- and 12.5-born PCs in the left E14.5 cerebellum. $\boldsymbol{E}$, Schematic of separation and migration of c-I lineage clusters (blue, green and red) at E17.5. Gray clusters are non-c-I-lineage clusters that separate the green clusters into the anterior and posterior parts and separate the blue cluster into the anterior and posterior parts later. $\boldsymbol{F}$, Mapping of the E10.5-PC-sparse areas that were considered to belong to the lineage of the c-I cluster mapped the unfolded scheme of the entire cerebellar cortex with the zebrin striped pattern (Sarpong et al., 2018). Projections of the B module and C1/C3/D0 modules are shown schematically. Abbreviations: 1+, 1and so on, aldolase C (zebrin) stripes 1+, 1- and so on; I-X, lobules I-X; a-c, sublobules a-c (as in IXa-b); AIN, anterior interposed nucleus; B, C1, C3, D: modules B, C1, C3 and D0; C, caudal; Cop, copula pyramidis; Cr I, crus I; Cr II, crus II; D, dorsal; FI, flocculus; ha4, ia1-2, ia3-5, ic1-2, ic3, ip1-2, it2, it3, va2-4 names of E17.5 PC clusters; L, lateral; LVN, lateral vestibular nucleus; M, medial; M1, primary motor cortex; Par, paramedian lobule; PFI, paraflocculus; R, rostral; Sim, simple lobule; V, ventral. 
Table 5: Afferent and efferent connections of the cerebellar modules that were derived from the c-I lineage clusters.

\begin{tabular}{|c|c|c|c|c|c|c|}
\hline E14.5 PC cluster & Birthdate of PCs & E17.5 PC cluster & Adult zebrin stripe(s) & Module & $1 \mathrm{O}^{1}$ & $\mathrm{DCN}^{2}$ \\
\hline \multirow[t]{6}{*}{ C-I } & E11.5, E12.5 & va2-4 & 2- (lateral) and b+ in I-V & $\mathrm{B}$ & dDAO & LVN \\
\hline & & ip1-2 & $4-, f+, f-, e 1+$ in Cop & $\mathrm{C} 1$ & vDAO & AIN \\
\hline & & ia1-2 & $b-, 3+$ in $I-V$ & C1 & vDAO & AIN \\
\hline & & ia3-5 (?) & $3-, 3 b+, 3 b-$ in III, IV-V & C1 & vDAO & AIN \\
\hline & & it3 & $\begin{array}{l}\text { 4-//5- (medial) in III, IV-V, Sim, } \\
\text { Cr I, Cr II, Par }\end{array}$ & C3 & vDAO, DM & AIN \\
\hline & & ha4 & $5-$ in IV-V & Do & DM & AIN \\
\hline
\end{tabular}

${ }_{1}^{1}$ Inferior olive subarea that project to PCs in the stripe (Sarpong et al., 2018; Sugihara and Shinoda, 2004).

${ }^{2}$ Cerebellar nucleus subarea that is innervated by PCs in the stripe (Sugihara et al., 2009).

Abbreviations: I-V, lobules I-V; III, lobule III; IV-V, lobule IV-V; AIN, anterior interposed nucleus; Cop, copula pyramidis; Cr I, crus II; Cr II, crus II; DCN, deep cerebellar nucleus; dDAO, dorsal fold of the dorsal accessory olive; DM dorsomedial subnucleus; IO, inferior olive; LVN, lateral vestibular nucleus; Par, paramedian lobule; Sim, simple lobule; vDAO, ventral fold of the dorsal accessory olive.

clusters named c-I differentiated into several zebrin stripes that belong to the $\mathrm{C} 1 / \mathrm{C} 3$ module in the anterior and posterior lobules. The results supported our hypothesis that the rearrangement of embryonic PC clusters shapes the compartmentalization of the adult cerebellar cortex which may underlie its modular organization and the dual somatotopy.

\section{Cerebellar compartmental organization originate from the differentiation of early PC clusters}

The present study revealed a progressive change in the compartmental organization in the embryonic cerebellum. The number of PC clusters recognized by the marker molecule expression profile increased from nine at E14.5 to 37 at E17.5. This increase was accompanied by a significant change in the spatial arrangement of PC clusters between E14.5 and E17.5, as demonstrated in the separation and migration of the c-I lineage clusters in the present study. Since the clustered compartments of $\mathrm{PCs}$ in the late embryonic stage (E17.5 in mice) are mostly comparable to the striped compartments of PCs in the adult (Fujita et al., 2012), our clarification of the compartmental differentiation in the period before E17.5 would lead to a better understanding (see below, "Developmental origin of the dual representation of the somatosensorimotor function in the cerebellum") of adult cerebellar compartmentalization. Compartmental organization at stages earlier than E14.5 was beyond the scope of the present study.

Concerning marker molecules used in this study, we speculate that transcription factors FoxP2 and Corl2 may control the expression of compartment-specific molecules, and adhesion molecule Pcdh10 and receptor tyrosine kinase EphA4 may be involved in cluster formation and cellto-cell connection between highly-expressing neurons (Vibulyaseck et al., 2017; Sarpong et al, 2018). However, the functional significance of these marker molecules in the development of PC compartmentalization has not been fully clarified. Nevertheless, differences in their expression levels among PCs were useful to detect trackable PC clusters in the present study. Based on different expression intensities of these marker molecules, we distinguished PC clusters in the entire cerebellum in embryonic dates between E14.5 and E17.5 (Figs. 2-5, Table 2)

The PC cluster organization identified in our study conformed to the PC organization that was identified based on gene expression profiling analysis at E14.5 (Wizeman et al., 2019), except for the Nrgn-positive cluster. The Nrgn -positive cluster, which is located widely above the ventricular zone and under other clusters (Wizeman et al., 2019), was not recognized as a cluster in our present study. Nrgn mRNA is expressed in neuro-progenitor and immature neurons in cerebral cortical culture (Nazir et al., 2018). Therefore, there is a possibility that Nrgn-positive cluster of Wizeman et al. (2019) represents newly-born $\mathrm{PCs}$ that are joining one of the other clusters. Besides, six of our nine E14.5 PC clusters m, c-m, c-I, d, ml, and I corresponded to clusters Ebf, Ebf/Calb1, Nrgn/Calb1, Ebf/ Dab1-dorsal, Ebf/Dab1-ventral and Foxp1/Dab1 in Fig. $4 H$ of Wizeman et al. (2019), respectively. It would mean that our PC clusters defined by marker molecule expression and PC birthdate and PC clusters defined by gene expression profiling make one-to-one correspondence, except for the Nrgn-positive cluster (above), at the caudal level of the cerebellum. The correspondence of three of our nine E14.5 clusters (dl, rdl and $\mathrm{vl}$ ) was not known because they were located at the more rostral level than the section shown in Wizeman et al. (2019). As a whole, the high consistency between our E14.5 cluster recognition and PC gene expression profiling support the cluster assembly that we recognized here represents not an arbitrarily defined structure but an essential organization of the E14.5 cerebellum.

\section{Birthdate-specific neuronal labeling to track the lineage of early PC compartments}

Tracing the lineage of early PC clusters in the embryonic cerebellum by birthdate-specific labeling of PCs was the essential method in this study. Since PCs of different birthdates are distributed heterogeneously in different PC compartments (Hashimoto and Mikoshiba, 2003; Namba et al., 2011), birthdate-specific labeling of PCs can be a useful experimental tool to track the lineage of PC clusters. Indeed, the original birthday-specific labeling with the asc/1 gene CreER-LoxP system showed that PCs labeled by tamoxifen given at E10.5, E11.5 and E12.5 are distributed differently in the adult cerebellum (Sudarov et al., 2011). Such a birthdate-specific labeling system is technically more accessible and appears more sensitive than previously established birthdate-specific labeling methods (systemic injection of 5-bromo-2'-deoxyuridine, BrdU; systemic injection of tritium-labeled thymidine, 
Altman and Bayer, 1985; or in-utero ventricular injection of replication-defective adenoviral vector, Hashimoto and Mikoshiba, 2004; Namba et al., 2011). G2A mice, in which the Neurog2 gene is targeted, allowed us to efficiently produce specimens with varying tamoxifen injection dates and survival periods in the present study. The labeling pattern of birthdate-specific PCs in the adult cerebellum in the present study was similar to, but not the same as, that reported with adenoviral vector labeling (Namba et al., 2011). For example, E11.5-born PCs were observed in most of the embryonic PC clusters and the majority of adult zebrin stripes in G2A mice (the present study and Zhang et al., 2020), they were distributed in a smaller number of PC compartments in adenoviral vector labeling. Such discrepancies may be due to some differences in the timing (e.g. different cell cycle points, different durations/efficiencies of labeling activity, etc.) of labeling differentiating neurons between different methods.

\section{Developmental origin of the dual representation of the somatosensorimotor function in the cerebellum}

The birthdate-tagging method employed in the present study revealed that the anteroposteriorly- and mediolaterally-separated stripes belonging to the somatosensorimotor C1/C3 modules originate mostly from the C-I cluster at E14.5, which is composed of E11.5- and E12.5 born PCs (Fig. 10C-F). The anteroposterior separation of the C1 module (the medial part of the C1/C3 module) occurred in the medial c-I lineage cluster at E15.5. Besides, the anteroposterior separation of the C3 module (the lateral part of the $\mathrm{C} 1 / \mathrm{C} 3$ module) occurs in the early postnatal period in the cluster which originates from the lateral c-l lineage cluster by the lateral migration of the $\mathrm{ml}$ lineage cluster (Fig. 10E, it3 cluster to be separated anteroposteriorly by ic5/it2 cluster) at P0-P1 as shown previously (Fujita et al., 2012). Although the timing is different, the anatomical process of anteroposterior separation seems similar between these two modules. To further understand this differentiation process, causal mechanisms that induce rostrocaudal separation of c-l lineage clusters are to be studied. The rostro-caudal link has been generally observed in axonal projections in compartments in paravermal and hemispheric modules (Sugihara and Shinoda, 2004; Fujita and Sugihara, 2013). We could not observe evidence of the anteroposterior separation of the B module or the most medial Corl2-strongly positive c-I lineage cluster, as well as evidence of the anteroposterior separation of the DO module or the most lateral c-I lineage cluster during the embryonic period in the present study, either.

Purkinje cells themselves play an essential role in the formation of the topographic afferent and efferent circuits (Sillitoe et al., 2009, 2010; White et al., 2014). The PC axonal projection and the olivocerebellar projection are directly linked to the compartmental or modular organization of the cerebellar cortex (Sugihara and Shinoda, 2004; Sugihara et al., 2009; Cerminara et al., 2013, Fig. 10F, Table 5). Single climbing fiber axons typically branch rostrocaudally and innervate both the anterior and posterior parts of the same module (Sugihara et al., 2001; Fujita and Sugihara, 2013). Axonal projections of PCs in the anterior and posterior parts of the same module converge on the same small area in the cerebellar nucleus (Sugihara et al., 2009). Such a rostrocaudal relationship in axonal projections is understandable by supposing the same axonal guidance cues (Sillitoe et al., 2009,2010 ) expressed by the pair of rostral and caudal PC clusters that originated from the same early cluster. Since PC compartments are topographically connected with subareas of the cerebellar nuclei and inferior olive (Ruigrok et al., 2015), the development of the cerebellar modules may depend on the concurrent development of the compartments in the cerebellar nuclei and inferior olive. Indeed, a genetically-induced defect in the developing cerebellar nuclei produces malformation of the cerebellar cortex (Willett et al., 2019). However, the development of compartments of the cerebellar nuclei or inferior olive has not been clarified yet to the level comparable to the fine compartmentalization shown in the cerebellar cortex (Fujita et al., 2012, 2020).

The mossy fiber projection, which is the main source of afferent axons to the cerebellar cortex, is not as tightly linked to PC compartments as PC axons or olivocerebellar axons (Quy et al., 2011; Biswas et al., 2019; Luo et al. 2020). However, because early mossy fibers initially target PCs (Kalinovsky et al., 2011), the early PC cluster organization may affect the mossy fiber projection pattern. Indeed, single mossy fibers often show branching to the anterior and posterior cerebellums (Biswas et al., 2019), which is similar to the anteroposterior separation of PC clusters shown in the present study. As a whole, the present study showed that the spatiotemporal differentiation process of early $\mathrm{PC}$ compartmentalization underlies the anteroposterior dual positioning of somatotopic areas in the cerebellar cortex (Snider, 1950; Stoodley et al., 2012; Guell et al., 2018), one of the most peculiar characteristics of cerebellar functional localization.

The present results propose a hypothesis about the general origin of the cerebellar compartmentalization: adult cerebellar compartments that share similar molecular expression profiles, axonal projections, and functional localization may generally originate from a common early $\mathrm{PC}$ compartment in cerebellar development. Besides the C1/C3 modules, our previous finding that the $\mathrm{ml}$ cluster at E14.5 became zebrin stripe $4+/ / 5+(4+$ in the anterior cerebellum and $5+$ in the posterior cerebellum), or the $\mathrm{C} 2$ module (Vibulyaseck et al., 2017) supports this hypothesis. However, the experimental results were not as simple. For example, the most medial part of the c-l cluster showed an increase in expression of Corl2 and was distinguished from the rest of the c-I cluster at E15.5 (Fig. 9A). It became stripes $2-$ and $b+$, forming the $B$ module, which projects to the lateral vestibular nucleus (Sugihara et al., 2009) for the control of posture and anti-gravity through the lateral vestibulospinal projection (Voogd, 2016). The axonal projection and function of the $\mathrm{B}$ module is distinct from those of the $\mathrm{C} 1 / \mathrm{C} 3$ module. The $\mathrm{B}$ module not only occupies a substantial area in the anterior cerebellum but also exists in the posterior cerebellum, in a small lateral vermal area of lobule VIII (stripe 4-, Fig. 10B4; Sugihara et al., 2009). The origin of this caudal B module was not clarified in the present study. The centrolateral part of the c-I cluster formed zebrin stripes 4-//5- (4- in the anterior 
cerebellum and 5 - in the posterior cerebellum, Fig. 10F) or the C3 module. The separation of stripe 4-//5- into medial and lateral substripes has been reported in our analysis of the PC birthdates in the adult cerebellar cortex (Zhang et al., 2020). It was noticeable that only the medial substripe of $4-/ / 5$ - originated from the c-l cluster. Consequently, the lateral substripe of $4-/ / 5$ - is supposed to originate from a different cluster at E14.5. The most lateral part of the c-I cluster formed a small part of zebrin-negative stripe 5-//6or the D0 module (Sugihara and Shinoda, 2004). The D0 module is the somatosensorimotor module akin to the C1/C3 modules and containing the area involved in the eye-blinking reflex (Attwell et al., 2001). According to the present results, most parts of the D0 module including the entire caudal parts of the DO module in the posterior cerebellum, seem to originate from different clusters. Thus, several questions remain regarding functional domains delineated by embryonic clusters and adult striped organization. The relationship between the early PC compartmentalization and the mossy fiber projection pattern is also to be studied.

\section{References}

Altman J, Bayer SA (1985) Embryonic development of the rat cerebellum. I. delineation of the cerebellar primordium and early cell movements. J Comp Neurol 231:1-26.

Apps R, Hawkes R (2009) Cerebellar cortical organization: A onemap hypothesis. Nature Reviews Neuroscience 10:670-681.

Attwell PJ, Rahman S, Yeo CH (2001) Acquisition of eyeblink conditioning is critically dependent on normal function in cerebellar cortical lobule HVI. Journal of Neuroscience 21:5715-5722.

Biswas MS, Luo Y, Sarpong GA, Sugihara I (2019) Divergent projections of single pontocerebellar axons to multiple cerebellar lobules in the mouse. J Comp Neurol 527:1966-1985.

Brochu G, Maler L, Hawkes R (1990) Zebrin II: A polypeptide antigen expressed selectively by Purkinje cells reveals compartments in rat and fish cerebellum. J Comp Neurol (United States) 291:538552.

Carter RA, Bihannic L, Rosencrance C, Hadley JL, Tong Y, Phoenix TN, Natarajan S, Easton J, Northcott PA, Gawad C (2018) A singlecell transcriptional atlas of the developing murine cerebellum. Current Biology 28:2910-2920. e2.

Cerminara NL, Aoki H, Loft M, Sugihara I, Apps R (2013) Structural basis of cerebellar microcircuits in the rat. Journal of Neuroscience 33:16427-16442.

Ekerot C, Garwicz M, Jörntell H (1997) The control of forelimb movements by intermediate cerebellum. In: Progress in brain research The control of forelimb movements by intermediate cerebellum. pp 423-429. Elsevier.

Florio M, Leto K, Muzio L, Tinterri A, Badaloni A, Croci L, Zordan P, Barili V, Albieri I, Guillemot F (2012) Neurogenin 2 regulates progenitor cell-cycle progression and Purkinje cell dendritogenesis in cerebellar development. Development 139:2308-2320.

Fujita H, Aoki H, Ajioka I, Yamazaki M, Abe M, Oh-Nishi A, Sakimura K, Sugihara I (2014) Detailed expression pattern of aldolase C (aldoc) in the cerebellum, retina and other areas of the CNS studied in aldoc-venus knock-in mice. PLoS One (United States) 9: e86679.

Fujita H, Sugihara I (2013) Branching patterns of olivocerebellar axons in relation to the compartmental organization of the cerebellum. Frontiers in Neural Circuits 7:3.

Fujita H, Morita N, Furuichi T, Sugihara I (2012) Clustered fine compartmentalization of the mouse embryonic cerebellar cortex and its rearrangement into the postnatal striped configuration. J Neurosci 32:15688-15703.
Fujita H, Kodama T, du Lac S (2020) Modular output circuits of the fastigial nucleus for diverse motor and nonmotor functions of the cerebellar vermis. eLife 9:e58613.

Goffinet AM (1983) The embryonic development of the cerebellum in normal and reeler mutant mice. Anat Embryol 168:73-86.

Guell X, Schmahmann JD, Gabrieli JD, Ghosh SS (2018) Functional gradients of the cerebellum. Elife 7:e36652.

Hashimoto M, Mikoshiba K (2004) Neuronal birthdate-specific gene transfer with adenoviral vectors. Journal of Neuroscience 24:286296.

Hashimoto M, Mikoshiba K (2003) Mediolateral compartmentalization of the cerebellum is determined on the "birth date" of Purkinje cells. Journal of Neuroscience 23:11342-11351.

Hirata T, Shioi G, Abe T, Kiyonari H, Kato S, Kobayashi K, Mori K, Kawasaki T (2019) A novel birthdate-labeling method reveals segregated parallel projections of mitral and external tufted cells in the main olfactory system. eNeuro 6:6.

Horn KM, Pong M, Gibson AR (2010) Functional relations of cerebellar modules of the cat. Journal of Neuroscience 30:9411-9423.

Kalinovsky A, Boukhtouche F, Blazeski R, Bornmann C, Suzuki N, Mason CA, Scheiffele P (2011) Development of axon-target specificity of ponto-cerebellar afferents. PLoS Biology 9:2.

Korneliussen HK (1968) On the ontogenetic development of the cerebellum (nuclei, fissures, and cortex) of the rat, with special reference to regional variations in corticogenesis. J Hirnforsch 10:379.

Larouche M, Che PM, Hawkes R (2006) Neurogranin expression identifies a novel array of Purkinje cell parasagittal stripes during mouse cerebellar development. J Comp Neurol 494:215-227.

Low AY, Thanawalla AR, Yip AK, Kim J, Wong KL, Tantra M, Augustine GJ, Chen Al (2018) Precision of discrete and rhythmic forelimb movements requires a distinct neuronal subpopulation in the interposed anterior nucleus. Cell Reports 22:2322-2333.

Luo Y, Onozato T, Wu X, Sasamura K, Sakimura K, Sugihara I (2020) Dense projection of Stilling's nucleus spinocerebellar axons that convey tail proprioception to the midline area in lobule VIII of the mouse cerebellum. Brain Struct Funct 225:621-638.

Luo Y, Patel RP, Sarpong GA, Sasamura K, Sugihara I (2018) Single axonal morphology and termination to cerebellar aldolase $C$ stripes characterize distinct spinocerebellar projection systems originating from the thoracic spinal cord in the mouse. J Comp Neurol 526:681-706.

Manni E, Petrosini L (2004) A century of cerebellar somatotopy: a debated representation. Nat Rev Neurosci 5:241-249.

Millen KJ, Hui C, Joyner AL (1995) A role for en-2 and other murine homologues of drosophila segment polarity genes in regulating positional information in the developing cerebellum. Development 121:3935-3945.

Minaki Y, Nakatani T, Mizuhara E, Inoue T, Ono Y (2008) Identification of a novel transcriptional corepressor, Corl2, as a cerebellar Purkinje cell-selective marker. Gene Expression Patterns 8:418-423.

Namba K, Sugihara I, Hashimoto M (2011) Close correlation between the birth date of Purkinje cells and the longitudinal compartmentalization of the mouse adult cerebellum. J Comp Neurol 519:25942614.

Nazir FH, Becker B, Brinkmalm A, Höglund K, Sandelius Å, Bergström P, Satir TM, Öhrfelt A, Blennow K, Agholme L (2018) Expression and secretion of synaptic proteins during stem cell differentiation to cortical neurons. Neurochem Int 121:38-49.

Oberdick J, Schilling K, Smeyne RJ, Corbin JG, Bocchiaro C, Morgan JI (1993) Control of segment-like patterns of gene expression in the mouse cerebellum. Neuron 10:1007-1018.

Pijpers A, Winkelman BH, Bronsing R, Ruigrok TJ (2008) Selective impairment of the cerebellar $\mathrm{C} 1$ module involved in rat hind limb control reduces step-dependent modulation of cutaneous reflexes. J Neurosci 28:2179-2189.

Quy PN, Fujita H, Sakamoto Y, Na J, Sugihara I (2011) Projection patterns of single mossy fiber axons originating from the dorsal column nuclei mapped on the aldolase $\mathrm{C}$ compartments in the rat cerebellar cortex. J Comp Neurol 519:874-899. 
Ruigrok TJ, Sillitoe RV, Voogd J (2015) Cerebellum and cerebellar connections. In: Paxinos G. ed, The Rat Nervous System. 4th ed. pp 133-205. Amsterdam: Elsevier Academic Press.

Sarpong GA, Vibulyaseck S, Luo Y, Biswas MS, Fujita H, Hirano S, Sugihara I (2018) Cerebellar modules in the olivo-cortico-nuclear loop demarcated by pcdh10 expression in the adult mouse. $\mathrm{J}$ Comp Neurol 526:2406-2427.

Sillitoe RV, Joyner AL (2007) Morphology, molecular codes, and circuitry produce the three-dimensional complexity of the cerebellum. Annu Rev Cell Dev Biol 23:549-577.

Sillitoe RV, Vogel MW, Joyner AL (2010) Engrailed homeobox genes regulate establishment of the cerebellar afferent circuit map. Journal of Neuroscience 30:10015-10024.

Sillitoe RV, Gopal N, Joyner AL (2009) Embryonic origins of Zebrinll parasagittal stripes and establishment of topographic Purkinje cell projections. Neuroscience 162:574-588.

Smeyne RJ, Oberdick J, Schilling K, Berrebi AS, Mugnaini E, Morgan Jl (1991) Dynamic organization of developing Purkinje cells revealed by transgene expression. Science 254:719-721.

Snider RS (1950) Recent contributions to the anatomy and physiology of the cerebellum. Archives of Neurology \& Psychiatry 64:196219.

Stoodley CJ, Valera EM, Schmahmann JD (2012) Functional topography of the cerebellum for motor and cognitive tasks: An fMRI study. Neuroimage 59:1560-1570.

Sudarov A, Turnbull RK, Kim EJ, Lebel-Potter M, Guillemot F, Joyner AL (2011) Ascl1 genetics reveals insights into cerebellum local circuit assembly. Journal of Neuroscience 31:11055-11069.

Sugihara I, Shinoda Y (2004) Molecular, topographic, and functional organization of the cerebellar cortex: A study with combined aldolase $\mathrm{C}$ and olivocerebellar labeling. Journal of Neuroscience 24:8771-8785.

Sugihara I, Wu HS, Shinoda Y (2001) The entire trajectories of single olivocerebellar axons in the cerebellar cortex and their contribution to cerebellar compartmentalization. J Neurosci 21:7715-7723.

Sugihara I, Fujita H, Na J, Quy PN, Li B, Ikeda D (2009) Projection of reconstructed single Purkinje cell axons in relation to the cortical and nuclear aldolase $\mathrm{C}$ compartments of the rat cerebellum. $\mathrm{J}$ Comp Neurol 512:282-304.
Thickbroom GW, Byrnes ML, Mastaglia FL (2003) Dual representation of the hand in the cerebellum: activation with voluntary and passive finger movement. Neurolmage 18:670-674.

Vibulyaseck S, Fujita H, Luo Y, Tran AK, Oh-Nishi A, Ono Y, Hirano S, Sugihara I (2017) Spatial rearrangement of Purkinje cell subsets forms the transverse and longitudinal compartmentalization in the mouse embryonic cerebellum. J Comp Neurol 525:2971-2990.

Voogd J, Glickstein M (1998) The anatomy of the cerebellum. Trends Neurosci (England) 21:370-375.

Voogd J (2016) Deiters' nucleus. its role in cerebellar ideogenesis. The Cerebellum 15:54-66.

Welker W (1987) Spatial Organization of Somatosensory Projections to Granule Cell Cerebellar Cortex: Functional and Connectional Implications of Fractured Somatotopy (Summary of Wisconsin Studies). In: New Concepts in Cerebellar Neurobiology, pp 239280. New York: Alan R Liss.

White JJ, Arancillo M, Stay TL, George-Jones NA, Levy SL, Heck DH, Sillitoe RV (2014) Cerebellar zonal patterning relies on Purkinje cell neurotransmission. J Neurosci 34(24):8231-8245.

Willett RT, Bayin NS, Lee AS, Krishnamurthy A, Wojcinski A, Lao Z, Stephen D, Rosello-Diez A, Dauber-Decker KL, Orvis GD, Wu Z, Tessier-Lavigne M, Joyner AL (2019) Cerebellar nuclei excitatory neurons regulate developmental scaling of presynaptic Purkinje cell number and organ growth. Elife 8:e50617.

Wilson SL, Kalinovsky A, Orvis GD, Joyner AL (2011) Spatially restricted and developmentally dynamic expression of engrailed genes in multiple cerebellar cell types. The Cerebellum 10:356372.

Wizeman JW, Guo Q, Wilion EM, Li JY (2019) Specification of diverse cell types during early neurogenesis of the mouse cerebellum. eLife 8:e42388.

Zhang J, Tran-Anh K, Hirata T, Sugihara I (2020) Striped distribution pattern of Purkinje cells of different birthdates in the mouse cerebellar cortex studied with the Neurog2-CreER transgenic line. Neuroscience, in press.

Zordan P, Croci L, Hawkes R, Consalez GG (2008) Comparative analysis of proneural gene expression in the embryonic cerebellum. Developmental Dynamics: An Official Publication of the American Association of Anatomists 237:1726-1735. 